\title{
Feasibility of Determining the Composition of Planetary Ices by Far Infrared Observations: Application to Martian Cloud and Surface Ices
}

\author{
Brian R. Johnson and Sushil K. Atreya \\ Department of Atmospheric, Oceanic and Space Sciences, University of Michigan, Ann Arbor, Michigan 48109-2143 \\ E-mail: bjohnson@ncar.ucar.edu
}

Received August 1, 1994; revised July 14, 1995

Ices in the atmospheres and on the surfaces of planets and moons are thought to play important roles in the evolution and stability of, and in radiative transfer in, planetary atmospheres. In this paper, the capability of far-infrared spectral observations to determine the composition and characteristics of planetary ices is investigated with particular application to martian $\mathrm{H}_{2} \mathrm{O}$ and $\mathrm{CO}_{2}$ ices. Thin film transmission spectra of crystalline (Ic) and amorphous $\mathrm{H}_{2} \mathrm{O}$ ice and crystalline $\mathrm{CO}_{2}$ ice were measured using a Fourier transform spectrometer. The far-infrared refractive indices of these ices at temperatures from 77 to $150 \mathrm{~K}$ over the spectral range 50 to $500 \mathrm{~cm}^{-1}$ were derived. These data are in generally good agreement with previously published indices.

The refractive index data were incorporated into a radiative transfer model used to study the far-infrared properties of cloud and surface ices on Mars. Typical mid-latitude $\mathrm{H}_{2} \mathrm{O}$ ice clouds on Mars have vertical far-infrared optical depths on the order of $10^{-4}$, precluding their detection using an earth-based remote sensing instrument. However, model calculations of polar condensates showed observable $\mathrm{H}_{2} \mathrm{O}$ ice cloud spectral features near the $225 \mathrm{~cm}^{-1}$ lattice absorption band. The presence of a $\mathrm{CO}_{2}$ ice haze lowered the apparent surface brightness temperature by 10 to $20 \mathrm{~K}$. Theoretical emission by $\mathrm{CO}_{2}$ frost showed strong spectral contrast in the surface brightness temperature of 20 to $30 \mathrm{~K}$ near the 66 and $110 \mathrm{~cm}^{-1}$ lattice bands in solid $\mathrm{CO}_{2}$. In the weakly absorbing inter-band region, $\mathrm{CO}_{2}$ frost emissivity varied from approximately 0.4 to 0.7 with the incorporation of small amounts $(0.1-1.0 \%)$ of dust or water ice. $\mathrm{H}_{2} \mathrm{O}$ frost exhibited poor spectral contrast with an emissivity close to unity.

The detection of polar hood condensates and of the presence of $\mathrm{H}_{2} \mathrm{O}$ ice and dust in the $\mathrm{CO}_{2}$ ice caps using an earth-based far-infrared instrument appears feasible, although a telescope with a mirror diameter on the order of 15 to $20 \mathrm{~m}$ or interferometric techniques are required to achieve adequate spatial resolution. It is speculated that these and other non-polar ices such as $\mathrm{N}_{2}$ and $\mathrm{CH}_{4}$, present in the outer solar system, will exhibit far-infrared spectral characteristics similar to $\mathrm{CO}_{2}$ ice, making their detection possible, but technically challenging. $\odot 1996 \mathrm{Aca}-$ demic Press, Inc.

\section{INTRODUCTION}

Ices are found in the atmospheres of many planets, as cloud and haze condensates, and on the surfaces of many of their satellites, particularly in the outer solar system. The principal ices in the solar system include $\mathrm{H}_{2} \mathrm{O}, \mathrm{CO}_{2}$, $\mathrm{CO}, \mathrm{CH}_{4}, \mathrm{C}_{2} \mathrm{H}_{2}, \mathrm{C}_{2} \mathrm{H}_{6}, \mathrm{~N}_{2}, \mathrm{NH}_{3}, \mathrm{~N}_{2} \mathrm{H}_{4}, \mathrm{HCN}$, and $\mathrm{NH}_{4} \mathrm{SH}$. Planetary ices are believed to play important roles in the origin, evolution, and stability of planetary atmospheres, in the planetary radiative energy balance, and in surfaceatmosphere interchange processes (see for example Atreya et al. 1989). For example, the formation of hydrazine and hydrocarbon ices and their subsequent transport to the deep atmosphere followed by pyrolysis is thought to play a key role in stabilizing ammonia and methane, respectively, against photolysis on the major planets (Atreya 1986, Hunten 1969 referred to the long-chain hydrocarbons as "gasoline"). Ices, including clathrate hydrates, are also considered to be the major source as well as sink of volatiles in the outer solar system and as such, they have probably played a crucial role in the formation and maintenance of the atmospheres on Titan, Triton, and Pluto.

On Mars, $\mathrm{H}_{2} \mathrm{O}$ and $\mathrm{CO}_{2}$ cloud and surface ices affect the radiative energy balance in the polar regions, strongly influencing the current climate. Incorporation of small amounts of dust or $\mathrm{H}_{2} \mathrm{O}$ ice in the polar caps modifies their albedo and emissivity, altering the radiative fluxes (Paige and Ingersoll 1985) and influencing the formation of the seasonal $\mathrm{CO}_{2}$ ice caps (James and North 1982). In addition, the seasonal condensation and sublimation of $\mathrm{CO}_{2}$ drives the condensation flow component of atmospheric circulation on Mars (Pollack et al. 1990). Atmospheric $\mathrm{H}_{2} \mathrm{O}$ ice or dust particles may also play a role in martian photochemistry. Recent homogeneous gas phase chemical modeling studies of the martian atmosphere show the rate of re-formation of atmospheric $\mathrm{CO}_{2}$ exceeds its photolytic destruction rate by about $40 \%$ (Atreya and $\mathrm{Gu} 1994$ ). To balance the formation and loss rates of $\mathrm{CO}_{2}$, Atreya and $\mathrm{Gu}$ suggested that some form of heterogeneous process 
involving the adsorption of key trace gases onto atmospheric dust or ice particles may be occurring. Because of the importance of ices in many atmospheric processes, it is highly desirable to measure their composition, including the presence of small amounts of contaminants in the ice, their spatial distribution, and their temporal variations.

The utility of far-infrared planetary observations has been largely unexplored primarily because the earth's atmospheric opacity in this spectral region precludes groundbased measurements. Infrared observations by Mariner 9 and Voyager spacecrafts were limited to frequencies above about $200 \mathrm{~cm}^{-1}$. Many planetary ices possess unique farinfrared spectral signatures that can be diagnostic of their composition. The far-infrared spectrum of ice is controlled to, a large degree, by lattice vibration bands which are a result of inter-molecular forces between bound molecules. This motion is a bound energy state, giving rise to translational or lattice modes usually having lower energy levels than typical gas phase rotational modes. The band centers of lattice modes in ices typically fall in the range from about 20 to $250 \mathrm{~cm}^{-1}$. The inter-molecular forces are generally much weaker than the intra-molecular bonding forces which dominate the near- and mid-infrared absorption mechanisms in both gases and solids. These strong intramolecular forces result in similar infrared spectra for gases and solids, with only minor shifts in the fundamental absorption band frequencies (Fink and Sill 1982). This leads to some ambiguity in interpreting near- and mid-infrared surface observations due to interfering atmospheric absorption.

Non-polar ices, such as $\mathrm{CO}_{2}$ or $\mathrm{N}_{2}$ ice, generally exhibit weaker absorption in the far infrared than ices containing strong $\mathrm{O}-\mathrm{H}$ bonds. This results in a frost with low emissivity over most of the far-infrared spectral region except near the lattice bands where the emissivity approaches unity due to the stronger in-band absorption. This kind of behavior leads to a relatively strong spectral contrast in emissivity between the absorption band and the nearby weakly absorbing inter-band region. In principle, it is possible to identify the composition of surface frosts and atmospheric condensates, and to estimate the relative abundances of individual constituents by observing the location and strengths of these far-infrared spectral features.

The spectral characteristics of the outgoing thermal emission from a planet depend on the emitting properties of the surface and on the distribution of gases and condensates in the atmosphere. Modeling the spectral characteristics of cloud and surface ices is central to addressing the feasibility of determining ice composition from remote sensing observations. Radiative transfer models are used to generate synthetic cloud and surface ice emission spectra which simulate the radiances measured by a remote sensing instrument. The strengths, widths, and locations of ice spectral features, and the spatial extent of clouds and surface ice will place fundamental requirements on any proposed earth-based far-infrared instrument. The interaction of thermal radiation with an ensemble of ice particles is dependent on particle composition, sizes, shapes, and concentrations and in addition for surface frosts, on the packing density (or porosity), temperature gradients, and largescale surface roughness. The fundamental parameter controlling the spectral behavior of ice particles is the single particle scattering albedo which is strongly dependent on the particle's complex refractive index, obtained from laboratory measurements.

Far-infrared laboratory transmission measurements of many planetary ices, such as $\mathrm{H}_{2} \mathrm{O}, \mathrm{CO}_{2}, \mathrm{CO}, \mathrm{C}_{2} \mathrm{H}_{2}, \mathrm{C}_{2} \mathrm{H}_{4}$, $\mathrm{C}_{2} \mathrm{H}_{6}, \mathrm{~N}_{2}$ and $\mathrm{NH}_{3}$ can be found in the literature (Bertie et al. 1969, Kuan 1969, Brown and King 1970, Ron and Schnepp 1967, Schwartz et al. 1971, Fink and Sill 1982, Sill et al. 1980). Generally, these laboratory measurements were concerned with assigning absorption bands, studying their internal structure, and comparing theoretically calculated lattice vibration intensities and frequencies with experimentally derived values. Large uncertainties in the absorption coefficients derived from these past measurements occur primarily due to the uncertainty in sample thickness and therefore the data are not well suited for accurate calculation of refractive indices. Recently, a number of laboratory measurements specifically designed to determine the near- and mid-infrared complex refractive indices of a wide range of planetary and interstellar ices have been conducted (see for example Khanna et al. 1988; Masterson and Khanna 1990, Khare et al. 1990; Salama et al. 1990, Pearl et al. 1991, Hansen 1993, Hudgins et al. 1993). However, little recent work has been conducted in the far-infrared region $\left(\nu<400 \mathrm{~cm}^{-1}\right)$ with the exception of $\mathrm{H}_{2} \mathrm{O}, \mathrm{CH}_{3} \mathrm{OH}$, and interstellar mixtures of $\mathrm{H}_{2} \mathrm{O}: \mathrm{CH}_{3} \mathrm{OH}: \mathrm{CO}: \mathrm{NH}_{3}$ reported by Hudgins et al. (1993).

In this paper, we discuss a general laboratory technique for measuring the far-infrared spectral transmission of planetary ices and present experimentally determined farinfrared absorption coefficients and refractive indices for $\mathrm{CO}_{2}$ and $\mathrm{H}_{2} \mathrm{O}$ ices. The refractive indices of these two ices were revisited both as a check of the experimental procedure and to make a quantitative assessment of the uncertainty in their far-infrared indices. The laboratory results reported here confirm earlier measurements and place a well-defined estimate of the uncertainty in the far-infrared refractive indices of $\mathrm{CO}_{2}$ and $\mathrm{H}_{2} \mathrm{O}$ ices. In addition, these ices have relevance to atmospheric and surface processes on Mars, and possibly on Triton and Pluto. The experimentally determined refractive indices of $\mathrm{CO}_{2}$ and $\mathrm{H}_{2} \mathrm{O}$ ices were incorporated into a detailed radiative transfer model to investigate the spectral properties of martian cloud and surface ices. Finally, we discuss the feasibility of detecting martian cloud and surface ices in the far infrared using an earth-based remote sensing 
instrument and we speculate on the feasibility of observing ices in the outer solar system.

\section{LABORATORY MEASUREMENTS}

Thin ice films were produced by condensing $\mathrm{CO}_{2}$ or $\mathrm{H}_{2} \mathrm{O}$ vapor onto a cold silicon substrate. Spectral transmission of the ice films was measured over the range from about 50 to $650 \mathrm{~cm}^{-1}$ using a Bomem DA8 Fourier transform spectrometer (FTS) equipped with Mylar beamsplitters and a deuterated triglycine sulfate (DTGS) detector. A high-pressure mercury source was used in the spectral range from 40 to $200 \mathrm{~cm}^{-1}$ and a Globar source was used for measurements made in the range from 200 to $700 \mathrm{~cm}^{-1}$. Due to the low output energy of these sources and the moderate sensitivity of the DTGS detector, several hundred spectra were added together to improve the signalto-noise ratio of the measurement. Even in doing this, noise was a significant source of uncertainty in the derived absorption coefficients and refractive indices.

A typical transmission measurement proceeded as follows. First, a reference transmission measurement was made of the blank substrate cooled to the deposition temperature of $77 \mathrm{~K}$. The cold finger and attached substrate were then rotated to face the gas injection port. Gas was allowed to flow through the injector at a carefully controlled rate, subsequently condensing onto the cold substrate. After deposition, the cold finger was rotated into the infrared beam and the ice transmission spectrum was measured. The ice film could be visually inspected by rotating the substrate to face a clear plastic window. An iterative Kramers-Kronig technique was used to determine the farinfrared complex refractive indices of $\mathrm{CO}_{2}$ and $\mathrm{H}_{2} \mathrm{O}$ ices from the measured transmission spectra. The details of this procedure are discussed in Appendix A of this paper. Nearand mid-infrared absorption data, needed for the Kramers-Kronig calculation, were obtained from the published compilation of Warren (1986) for $\mathrm{CO}_{2}$ ice and measurements made by Bertie et al. (1969) for $\mathrm{H}_{2} \mathrm{O}$ ice.

The ice samples were prepared in an Oxford CF1104 continuous flow vacuum cryostat. Optical access to the ice sample was provided by four orthogonal window ports (see Fig. 1). The two windows of the cryostat in the infrared beam were made of 2-mm-thick polyethylene. A gas injector was fitted into the side window port. The temperature of the cryostat cold finger was automatically controlled over the range from 77 to $300 \mathrm{~K}$ by an Oxford Intelligent Temperature Controller (ITC-4) and could be maintained to within $1.0 \mathrm{~K}$ of the set-point temperature. The substrate temperature was measured using a platinum resistive temperature device mounted onto the substrate holder, with an estimated error of $\pm 1.2 \mathrm{~K}$. A highly polished wedged silicon substrate was used because of its high thermal conductivity and low thermal expansion coefficient, making it

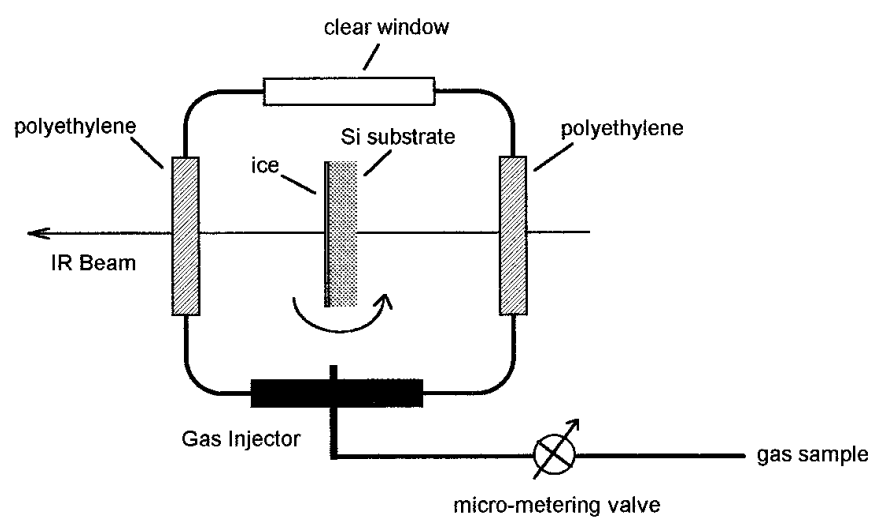

FIG. 1. Diagram of vacuum cryostat and sample holder used in farinfrared transmission measurements.

well suited for this application. A disadvantage to using silicon is the relatively high refractive index (measured to be 3.58 at $100 \mathrm{~K}$ and 3.62 at $290 \mathrm{~K}$ ), which results in a significant reflection loss of roughly $50 \%$ over the spectral region of interest. In addition, silicon exhibits temperaturedependent electronic conduction absorption loss below about $100 \mathrm{~cm}^{-1}$ and lattice band absorption near $610 \mathrm{~cm}^{-1}$. The silicon substrate transmission characteristics and the complex refractive index at $77 \mathrm{~K}$ were determined from laboratory measurements.

The $\mathrm{CO}_{2}$ gas sample was taken directly from a gas cylinder with a stated purity of $99.95 \%$ (research purity) from Matheson Gas Products and containing less than 2 ppm of $\mathrm{H}_{2} \mathrm{O}$. No further purification was made. For $\mathrm{H}_{2} \mathrm{O}$ ice deposition, deionized, distilled liquid water was degassed under vacuum and allowed to flow into a water ice cold trap. Three condensation-degassing cycles were performed to further purify the water vapor sample. The gas flow rate into the cryostat was precisely controlled by a micro-metering valve, with typical uncalibrated flow rates of roughly $5.2 \mathrm{ml} / \mathrm{hr}$ corresponding to a deposit rate of approximately $12 \mu \mathrm{m} / \mathrm{hr}$. The maximum $\mathrm{CO}_{2}$ film thickness obtainable was approximately $35 \mu \mathrm{m}$. The quality of the films for both $\mathrm{CO}_{2}$ and $\mathrm{H}_{2} \mathrm{O}$ ices were extremely sensitive to flow rate. Only $\mathrm{CO}_{2}$ ice transmission spectra exhibiting uniform interference fringes and minimal scattering effects were used in determining the refractive index. Films of only a few $\mu \mathrm{m}$ were necessary to adequately measure the absorption coefficient of $\mathrm{H}_{2} \mathrm{O}$ ice. Transmission measurements were made of crystalline $\mathrm{CO}_{2}$ ice in the temperature range from 80 to $120 \mathrm{~K}$ and of amorphous and cubic (Ic) water ice in the range from 77 to $150 \mathrm{~K}$. The results of the thin film transmission measurements and the determination of far-infrared refractive indices of $\mathrm{CO}_{2}$ and $\mathrm{H}_{2} \mathrm{O}$ ices are presented in Section 4.

It should be mentioned that deposition temperature also controls the solid phase characteristics and hence, the infra- 
red spectra of both $\mathrm{H}_{2} \mathrm{O}$ and $\mathrm{CO}_{2}$ ices. This is an important consideration when comparing measurements made by other investigators (e.g., Kuan 1969, Hudgins et al. 1993) at different deposition temperatures. At temperatures below about $30 \mathrm{~K}$ (well below the $77 \mathrm{~K}$ deposition temperatures used in our experiments), $\mathrm{CO}_{2}$ ice will form an amorphous film which when warmed to temperatures above about 50 K, will irreversibly change to crystalline ice (Falk 1987). Amorphous water ice is formed at deposition temperatures below about $140 \mathrm{~K}$. Warming the film to temperatures between 140 and $190 \mathrm{~K}$ results in a phase change to cubic (Ic) ice and at temperatures above $190 \mathrm{~K}$, hexagonal (Ih) ice is formed.

\section{MARTIAN CLOUD AND SURFACE ICES}

In this section, spacecraft observations of martian aerosols, clouds, and surface ices are briefly reviewed. The characteristics of cloud and surface ices inferred from these observation were used to develop aerosol, cloud and surface models describing particle composition, sizes, and vertical distribution for mid-latitude and polar conditions. These models provide basic inputs to the radiative transfer model. The solution to the radiative transfer equation using a line-by-line direct integration method coupled with a successive orders of scattering technique is discussed in Appendix B.

Atmospheric models for mid-latitude and polar region conditions were developed primarily based upon Mariner and Viking spacecraft observations. The atmospheric pressure at the Martian surface varies significantly with season from about 6 to 9 mbars. There are large diurnal variations in surface temperature (e.g., 180 to $270 \mathrm{~K}$ at $60^{\circ} \mathrm{N}$ latitude). Small temperature inversions are common in the boundary layer, particularly for winter polar latitudes. The temperature structure used in the polar atmospheric models was based on Lindal et al.'s (1979) analysis of Viking radio occultation measurements. The standard martian atmosphere volume mixing ratios were assumed for $\mathrm{CO}_{2}, \mathrm{~N}_{2}$, $\mathrm{Ar}, \mathrm{O}_{2}$, and $\mathrm{CO}$ (Owen et al. 1977). Water vapor is highly variable with location and season on Mars. The water vapor amount assumed in the mid-latitude model was the globally averaged column abundance of $10 \mathrm{pr} \mu \mathrm{m}$ (Jakosky and Farmer 1982; 1 precipitable micron $(\mathrm{pr} \mu \mathrm{m})=10^{-4} \mathrm{~g} \mathrm{~cm}^{-2}$ $\mathrm{H}_{2} \mathrm{O}=3.35 \times 10^{18} \mathrm{~cm}^{-2} \mathrm{H}_{2} \mathrm{O}$ or about $15 \mathrm{ppm}$ for uniformly mixed $\mathrm{H}_{2} \mathrm{O}$-vapor). In the polar wintertime, water vapor appears to be at or near its saturation vapor pressure at all altitudes (Jakosky 1985). Therefore, the water vapor profile in the polar atmospheric models was constrained to follow its saturation vapor pressure curve as defined by the Clausius-Clapeyron equation. The abundance of $\mathrm{O}_{3}$ is also variable with latitude and season peaking in the polar region near $57^{\circ} \mathrm{N}$, with a maximum measured ozone amount of $57 \mu \mathrm{m}$-atm over the polar hood during late winter and falling off rapidly with latitude to approximately 15 to $20 \mu \mathrm{m}$-atm at $80^{\circ} \mathrm{N}$ (Barth 1974, $1 \mu \mathrm{m}$-atm $=$ $2.687 \times 10^{15} \mathrm{~cm}^{-2}$ ). A value of $15 \mu \mathrm{m}$-atm of $\mathrm{O}_{3}$ was used in the winter polar atmospheric model calculations.

Thin detached hazes or discrete clouds composed of water ice particles are frequently observed in the midlatitudes and high latitudes on Mars. Mariner 9 IRIS limb spectra measured in the Tharsis Ridge region showed absorption features at 230 and $800 \mathrm{~cm}^{-1}$, indicating the presence of $\mathrm{H}_{2} \mathrm{O}$ ice clouds (Curran et al. 1973). Curran et al. estimated a mean particle size of $2 \mu \mathrm{m}$ and an integrated cloud mass of $5 \times 10^{-5} \mathrm{gm} / \mathrm{cm}^{2}$ by comparing the measured IRIS spectra with theoretical calculations. The location of these clouds near shield volcanoes suggest they were formed in the uplift of prevailing winds (Rossow 1978). Analysis of Viking Orbiter limb images by Kahn (1990) showed numerous discrete haze layers occurring between 20 and $80 \mathrm{~km}$. These hazes appeared to be composed of water ice with temperatures of 170 to $190 \mathrm{~K}$ at the condensation level and ice concentrations ranging from 0.0021 to $0.034 \mathrm{pr} \mu \mathrm{m}$. Kahn found that the ice particle sizes varied with condensation level from about 0.1 to a few $\mu \mathrm{m}$. The horizontal extent of these hazes can be a few hundred to $1000 \mathrm{~km}$. These detached hazes are persistent and Kahn suggests that they may be analogous to the earth's polar stratospheric clouds. Discrete layers of water ice clouds were also detected at several locations up to $50 \mathrm{~km}$ by spectrometers on the Phobos 2 spacecraft (Chassefierre et al. 1992). The vertical extent of these clouds was typically 3-6 km. The clouds were inferred to be composed of water ice because they formed at altitudes where the temperatures were above the frost point of $\mathrm{CO}_{2}$. Chassefièrre et al. derived an effective cloud particle radius of 0.15 to 0.85 $\mu \mathrm{m}$ with an effective variance of 0.2 from solar occultation measurements. The peak number densities of cloud particles were $\sim 1 \mathrm{~cm}^{-3}$.

During the polar winter, temperatures become cold enough for carbon dioxide to condense in the atmosphere, forming a polar hood beginning in the autumnal equinox. Atmospheric dust is also expected to be present in the polar hood. Observations by Mariner 9 showed the polar hood to be highly variable from day to day. Briggs and Leovy (1974) suggested that this variability was the result of the motion and decay of baroclinic waves. A variety of cloud types have been associated with the polar hood including lee wave clouds near the cap edge, probably composed of water ice and convective clouds, likely composed of $\mathrm{CO}_{2}$ ice. The water ice haze has a typical visible optical depth close to unity, equivalent to about 1-2 pr $\mu \mathrm{m}$ of water ice (Christensen and Zurek 1984). The haze is nearly always present during the annual retreat of the polar ice cap. Diffuse, low-lying hazes are prevalent poleward of about $60^{\circ} \mathrm{N}$ with a scale height of 3 to $4 \mathrm{~km}$ extending to $20 \mathrm{~km}$ (Pollack et al. 1977). These hazes form 
at atmospheric temperatures near the frost point of $\mathrm{CO}_{2}$ and their composition is inferred to be $\mathrm{CO}_{2}$ ice or a mixture of $\mathrm{CO}_{2}$ and $\mathrm{H}_{2} \mathrm{O}$ ices. $\mathrm{CO}_{2}$ ice particles are expected to grow quite large, approaching $25 \mu \mathrm{m}$, using water ice or dust particles as condensation nuclei. Also in the polar region, a cloud layer typically $4 \mathrm{~km}$ thick forming at about $15 \mathrm{~km}$ and a hazy cloud with a scale height close to the gas scale height up to about $20 \mathrm{~km}$ have been observed (Briggs and Leovy 1974).

Suspended dust is always present in the atmosphere, in varying amounts. Its presence has been detected by spacecraft and earth-based telescopic observations. From Viking observations, the typical sizes of suspended dust particles are on the order of a few $\mu \mathrm{m}$ and distributed in altitude from the surface to roughly 30 to $50 \mathrm{~km}$ with a scale height close to the gas scale height. The background or "clear-air" dust amounts correspond to visible optical depths of about 0.2 to 0.3 . For a "dusty" atmosphere during dust storm conditions, the optical depth increases to 4 or 5. Dust opacity in the polar regions is typically a factor of 2 or 3 lower than at other latitudes. Analysis of Mariner IRIS spectra by Conrath (1975) showed the atmospheric dust to be uniformly mixed to a height of about $35 \mathrm{~km}$. Above this level, the mixing ratio rapidly fell off with an effective top of the dust layer around $50 \mathrm{~km}$. From Viking observations, Pollack et al. (1977) derived a cross section weighted mean radius for dust aerosols of $2.5 \mu \mathrm{m}$ and a vertical number density profile of the form $n(z)=n_{0}$ $\exp \left(-z / H_{\mathrm{d}}\right)$ extending to about $30 \mathrm{~km}$. Toon et al. (1977) were able to derive dust properties from Mariner 9 IRIS observations during the dissipation phase of a global dust storm. Toon et al. found that a modified gamma size distribution with a mode radius of $0.4 \mu \mathrm{m}, \alpha=2$ and $\gamma=0.5$ produced the best fit to the measured spectrum.

In Toon et al.'s analysis, the optical properties of martian dust seemed to be well matched with those of a mixture of montmorillonite and basalt. However, a later comparison of earth-based visible and near-infrared telescopic spectra of Mars with laboratory reflectance spectra of several terrestrial materials showed palagonites to be the closest spectral analog to the martian dust (Singer 1982). Santee and Crisp (1993) analyzed Mariner 9 IRIS data using the spectral properties of palagonite in their analysis of Mars thermal structure and dust loading. They found palagonite to be a better match to IRIS spectra in the region 9 to $15 \mu \mathrm{m}$ than montmorillonite used in Toon et al.'s analysis, although they noted that Toon et al.'s analysis was for dusty conditions and the IRIS spectra they analyzed were for clear conditions. The far-infrared complex refractive indices of the various candidate dust materials, such as montmorillonite, basalt, and palagonites, are not readily available. Lacking more complete laboratory data for these materials, the refractive index of olivine was used to model the optical properties of both the martian regolith and atmospheric dust. Mukai and Koike (1990) derived the far-infrared index of refraction of olivine from laboratory measurements. The exact nature of the far-infrared optical properties of dust will not affect the general results or conclusions regarding the delectability of cloud and surface ices.

The exact composition of the martian regolith is still uncertain, although silicates and iron-rich materials are certain. Overall, the material is well matched by basalt with varying degrees of oxidation and grain sizes. The reddish color of martian soil is attributed to iron oxides. Palagonites are currently the best analog to explain visible and near-infrared reflectance spectra. Hematite and goethite are typical crystalline iron materials that are also likely to be present in the regolith (Soderblom 1993). In the case of modeling the martian regolith, olivine was also used to describe the optical properties of surface particles.

Surface ices or frosts are present in the polar regions on Mars. In the north polar region, there is a residual water ice cap mixed with large amounts of dust (Kieffer et al. 1976). Kieffer (1990) suggested that the residual cap is probably a mixture of fine dust and $\mathrm{H}_{2} \mathrm{O}$ ice grains with comparable sizes and abundance. The ice grain size is dependent on the age of the frost deposit, with older material consisting of larger grain sizes. Viking water vapor observations have shown an annual average decrease in the amount of atmospheric water vapor, leading to the conclusion that the north polar cap is currently in a net sublimation phase and the exposed surface ice is probably older ice with ice grain sizes $\sim 100 \mu \mathrm{m}$ or more (Kieffer 1990). In the southern hemisphere, the residual polar ice cap is composed of primarily carbon dioxide ice, or possibly a mixture of carbon dioxide ice and water ice. Analysis by Calvin (1990) of Mariner 7 near-infrared reflection spectra of the southern polar $\mathrm{CO}_{2}$ ice frost indicates that ice grain sizes are quite large, probably of the order of millimeters to centimeters. From Calvin's analysis, there are also indications that the $\mathrm{CO}_{2}$ frost is contaminated with $\mathrm{H}_{2} \mathrm{O}$ ice or dust, but more precise laboratory measurements of $\mathrm{CO}_{2}$ ice absorption are needed. The condensation of atmospheric $\mathrm{CO}_{2}$ in the polar winter forms a seasonal canopy of $\mathrm{CO}_{2}$ frost. Toon et al. (1980) estimated the volume mixing ratio of dust incorporated into the seasonal $\mathrm{CO}_{2}$ polar ice caps in the north to be approximately $10^{-3}$ and in the south to be approximately $10^{-4}$.

\section{EXPERIMENTAL AND MODELING RESULTS}

The results of the laboratory measurements and the theoretically calculated spectral characteristics of martian $\mathrm{H}_{2} \mathrm{O}$ and $\mathrm{CO}_{2}$ ices are presented in this section. Using the experimentally determined indices, theoretical emissivities of $\mathrm{H}_{2} \mathrm{O}$ and $\mathrm{CO}_{2}$ frosts are calculated using Hapke's emittance model (Hapke 1993). A detailed radiative transfer 

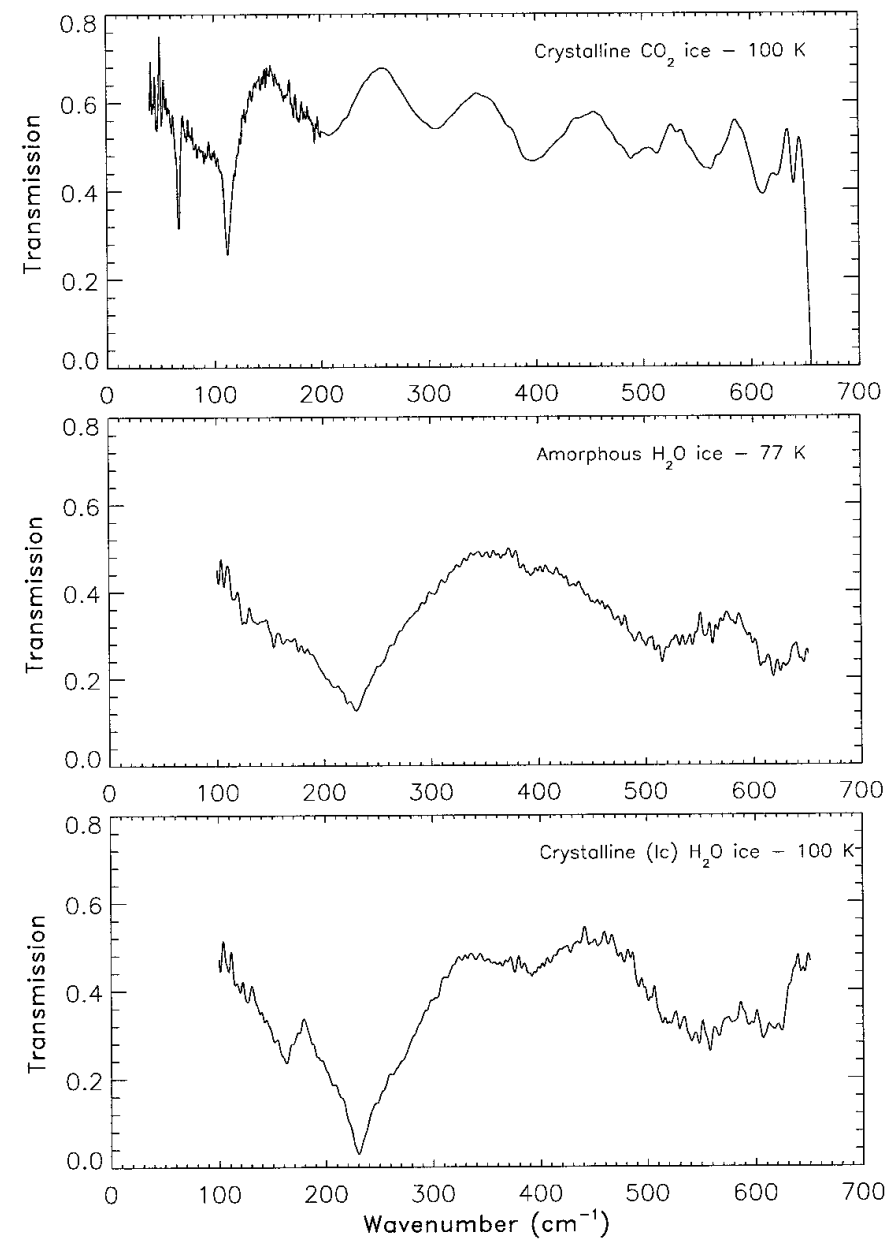

FIG. 2. Measured far-infrared transmission spectrum of a 34- $\mu \mathrm{m}$ thick $\mathrm{CO}_{2}$ ice film at $100 \mathrm{~K}$ (top panel), a $10-\mu \mathrm{m}$-thick amorphous water ice film at $77 \mathrm{~K}$ (middle panel), and a crystalline water ice (Ic) film at $100 \mathrm{~K}$, after being annealed at $150 \mathrm{~K}$ for $30 \mathrm{~min}$ (bottom panel).

model was used to calculate the far infrared spectra of martian cloud and surface ices. The results of the modeling study for mid-latitude water ice clouds are summarized and calculated polar cloud and surface ice spectra are presented.

\subsection{Refractive Indices}

The laboratory measurements are of fundamental importance in modeling the optical properties of ice particles. Several transmission measurements were made of crystalline $\mathrm{CO}_{2}$ ice, and of amorphous and crystalline water ice films of various film thickness ranging from a few $\mu \mathrm{m}$ to about $35 \mu \mathrm{m}$. Examples of these measurements are shown in Fig. 2. In the top panel of Fig. 2, the transmission spectrum of a $34-\mu \mathrm{m}$-thick $\mathrm{CO}_{2}$ ice film deposited at $100 \mathrm{~K}$ is shown. This curve is a composite of two separate measurements of the same ice film because it was necessary to change the Mylar beamsplitter and the infrared source to cover the spectral range. The transmission curve is spliced together at $200 \mathrm{~cm}^{-1}$ with a slight adjustment in the transmission level of the 200 to $700 \mathrm{~cm}^{-1}$ curve to provide a good match at this point. Two lattice absorption bands are clearly seen near 66 and $112 \mathrm{~cm}^{-1}$, measured with a spectral resolution of $1 \mathrm{~cm}^{-1}$ and averaging 600 scans together. Interference fringes, resulting from multiple reflection within the ice film, are seen in the spectral region above $150 \mathrm{~cm}^{-1}$, measured with a spectral resolution of $8 \mathrm{~cm}^{-1}$ and averaging 800 scans. The fundamental bending mode in solid $\mathrm{CO}_{2}$ is seen near $660 \mathrm{~cm}^{-1}$, which is opaque for this sample thickness. The weaker spectral feature near $638 \mathrm{~cm}^{-1}$ is due to the isotope ${ }^{16} \mathrm{O}-{ }^{13} \mathrm{C}-{ }^{16} \mathrm{O}$ (Falk 1987). The lattice vibration absorption band in the silicon substrate can be seen near $610 \mathrm{~cm}^{-1}$.

Transmission through a $10-\mu \mathrm{m}$-thick amorphous water ice film deposited at $77 \mathrm{~K}$ is shown in the middle panel of Fig. 2. The transmission spectrum shows a single broad lattice absorption peak near $230 \mathrm{~cm}^{-1}$. The water ice film was warmed to $150 \mathrm{~K}$ and allowed to anneal for $30 \mathrm{~min}$, forming crystalline ice. The substrate was then cooled to $100 \mathrm{~K}$ and the measured transmission spectrum is shown in the bottom panel of Fig. 2. The absorption peak near $230 \mathrm{~cm}^{-1}$ is much sharper in the crystalline film and a second absorption peak appears near $160 \mathrm{~cm}^{-1}$. The water ice measurements were made with a spectral resolution of 4 $\mathrm{cm}^{-1}$ with 800 scans averaged together. The lower spectral resolution was necessary to improve the signal-to-noise ratio of measurement.

The lattice absorption bands in ices show a marked temperature dependence, as discussed by Fink and Sill (1982). According to Fink and Sill, practically all ices show a sharpening of their infrared absorption features with decreasing temperature. The crystal lattice motions decrease with decreasing temperature, resulting in smaller perturbations of the vibrational energy states leading to longer lifetimes and producing narrower transitions. The locations of the lattice bands in solid $\mathrm{CO}_{2}$ shift to lower wavenumbers with increasing temperature and the width of the absorption bands broaden. The $112 \mathrm{~cm}^{-1}$ band shows a greater temperature sensitivity than does the $66 \mathrm{~cm}^{-1}$ band. The measured center frequencies (estimated using the full width at half-maximum values) for the two lattice bands of $\mathrm{CO}_{2}$ ice at 80,100 , and $120 \mathrm{~K}$ were $113.9,112.0$, and $110.3 \mathrm{~cm}^{-1}$ and $67.0,66.5$, and $66.2 \mathrm{~cm}^{-1}$, respectively. The absorption coefficient for $\mathrm{H}_{2} \mathrm{O}$ ice also shows a noticeable temperature dependence in addition to passing through a phase transition near $140 \mathrm{~K}$ which significantly alters the far-infrared spectrum. The measured location of the main absorption peak in $\mathrm{H}_{2} \mathrm{O}$ ice was $230 \mathrm{~cm}^{-1}$ for amorphous water ice at 77 $\mathrm{K}, 230.7 \mathrm{~cm}^{-1}$ for crystalline $\mathrm{H}_{2} \mathrm{O}$ ice at $100 \mathrm{~K}$, and 227 $\mathrm{cm}^{-1}$ for crystalline $\mathrm{H}_{2} \mathrm{O}$ ice at $150 \mathrm{~K}$. 
TABLE I

Measured Absorption Coefficients and Refractive Indices of Crystalline $\mathrm{CO}_{2}$ Ice

\begin{tabular}{|c|c|c|c|c|c|c|c|c|c|c|c|}
\hline \multicolumn{4}{|c|}{$80 \mathrm{~K}$} & \multicolumn{4}{|c|}{$100 \mathrm{~K}$} & \multicolumn{4}{|c|}{$120 \mathrm{~K}$} \\
\hline $\begin{array}{c}\nu \\
\left(\mathrm{cm}^{-1}\right)\end{array}$ & $\begin{array}{c}\alpha_{\mathrm{p}} \\
\left(\mathrm{cm}^{-1}\right)\end{array}$ & $n$ & $k$ & $\begin{array}{c}\nu \\
\left(\mathrm{cm}^{-1}\right)\end{array}$ & $\begin{array}{c}\alpha_{\mathrm{p}} \\
\left(\mathrm{cm}^{-1}\right)\end{array}$ & $n$ & $k$ & $\begin{array}{c}\nu \\
\left(\mathrm{cm}^{-1}\right)\end{array}$ & $\begin{array}{c}\alpha_{\mathrm{p}} \\
\left(\mathrm{cm}^{-1}\right)\end{array}$ & $n$ & $k$ \\
\hline 62.0 & 11.6 & 1.489 & 0.015 & 62.0 & 12.7 & 1.487 & 0.016 & 61.0 & 10.4 & 1.464 & 0.014 \\
\hline 63.0 & 17.6 & 1.501 & 0.022 & 63.0 & 20.1 & 1.500 & 0.025 & 62.0 & 15.1 & 1.470 & 0.019 \\
\hline 64.0 & 29.6 & 1.519 & 0.037 & 64.0 & 35.9 & 1.518 & 0.045 & 63.0 & 23.3 & 1.477 & 0.029 \\
\hline 64.5 & 40.1 & 1.530 & 0.050 & 64.5 & 50.6 & 1.529 & 0.062 & 63.5 & 29.6 & 1.480 & 0.037 \\
\hline 65.0 & 56.6 & 1.542 & 0.069 & 65.0 & 74.1 & 1.538 & 0.091 & 64.0 & 38.4 & 1.483 & 0.048 \\
\hline 65.5 & 83.1 & 1.552 & 0.101 & 65.5 & 110.9 & 1.538 & 0.135 & 64.5 & 50.1 & 1.483 & 0.062 \\
\hline 66.0 & 124.4 & 1.551 & 0.150 & 65.9 & 148.6 & 1.516 & 0.179 & 65.0 & 64.9 & 1.478 & 0.079 \\
\hline 66.3 & 155.9 & 1.536 & 0.187 & 66.3 & 179.3 & 1.465 & 0.215 & 65.4 & 77.4 & 1.468 & 0.094 \\
\hline 66.5 & 177.0 & 1.516 & 0.212 & 66.5 & 184.4 & 1.431 & 0.221 & 65.8 & 87.8 & 1.451 & 0.106 \\
\hline 67.0 & 206.1 & 1.452 & 0.245 & 66.9 & 168.3 & 1.367 & 0.200 & 66.2 & 92.2 & 1.429 & 0.111 \\
\hline 67.3 & 196.4 & 1.377 & 0.232 & 67.3 & 132.6 & 1.331 & 0.157 & 66.6 & 88.8 & 1.407 & 0.106 \\
\hline 67.7 & 159.2 & 1.328 & 0.187 & 67.7 & 98.0 & 1.321 & 0.115 & 67.0 & 79.4 & 1.390 & 0.094 \\
\hline 68.0 & 128.2 & 1.313 & 0.150 & 68.0 & 77.6 & 1.323 & 0.091 & 67.4 & 67.3 & 1.380 & 0.079 \\
\hline 68.5 & 87.0 & 1.312 & 0.101 & 68.5 & 53.8 & 1.332 & 0.063 & 68.0 & 50.3 & 1.374 & 0.059 \\
\hline 69.0 & 60.2 & 1.323 & 0.069 & 69.0 & 38.8 & 1.343 & 0.045 & 68.5 & 39.2 & 1.375 & 0.046 \\
\hline 69.5 & 43.4 & 1.335 & 0.050 & 69.5 & 29.1 & 1.353 & 0.033 & 69.0 & 30.9 & 1.378 & 0.036 \\
\hline 70.0 & 32.5 & 1.346 & 0.037 & 70.0 & 22.6 & 1.362 & 0.026 & 69.5 & 24.7 & 1.382 & 0.028 \\
\hline 71.0 & 20.1 & 1.363 & 0.022 & 71.0 & 14.8 & 1.375 & 0.017 & 70.0 & 20.2 & 1.386 & 0.023 \\
\hline 72.0 & 13.7 & 1.376 & 0.015 & 72.0 & 10.5 & 1.384 & 0.012 & 71.0 & 14.2 & 1.392 & 0.016 \\
\hline 106.0 & 24.9 & 1.472 & 0.019 & 104.0 & 24.5 & 1.462 & 0.019 & 101.0 & 20.2 & 1.450 & 0.016 \\
\hline 107.0 & 31.9 & 1.479 & 0.024 & 105.0 & 31.0 & 1.467 & 0.024 & 102.0 & 24.6 & 1.453 & 0.019 \\
\hline 108.0 & 42.2 & 1.487 & 0.031 & 106.0 & 40.3 & 1.473 & 0.030 & 103.0 & 30.3 & 1.456 & 0.023 \\
\hline 109.0 & 57.8 & 1.497 & 0.042 & 107.0 & 53.7 & 1.479 & 0.040 & 104.0 & 37.9 & 1.459 & 0.029 \\
\hline 110.0 & 82.4 & 1.508 & 0.060 & 108.0 & 73.6 & 1.485 & 0.054 & 105.0 & 48.2 & 1.462 & 0.037 \\
\hline 111.0 & 122.3 & 1.517 & 0.088 & 109.0 & 103.0 & 1.488 & 0.075 & 106.0 & 62.1 & 1.464 & 0.047 \\
\hline 112.0 & 184.8 & 1.515 & 0.131 & 110.0 & 143.8 & 1.483 & 0.104 & 107.0 & 80.3 & 1.463 & 0.060 \\
\hline 112.9 & 255.6 & 1.487 & 0.180 & 111.1 & 192.4 & 1.455 & 0.138 & 108.0 & 102.3 & 1.457 & 0.075 \\
\hline 113.4 & 287.5 & 1.455 & 0.202 & 111.6 & 206.9 & 1.433 & 0.148 & 109.4 & 131.1 & 1.436 & 0.095 \\
\hline 113.9 & 300.8 & 1.414 & 0.210 & 112.0 & 212.5 & 1.409 & 0.151 & 110.3 & 138.6 & 1.412 & 0.100 \\
\hline 114.4 & 290.1 & 1.373 & 0.202 & 112.6 & 204.3 & 1.385 & 0.144 & 111.4 & 130.4 & 1.389 & 0.093 \\
\hline 114.9 & 260.2 & 1.340 & 0.180 & 113.1 & 188.1 & 1.365 & 0.132 & 112.0 & 119.5 & 1.379 & 0.085 \\
\hline 116.0 & 176.7 & 1.310 & 0.121 & 114.0 & 149.0 & 1.345 & 0.104 & 113.0 & 97.8 & 1.368 & 0.069 \\
\hline 117.0 & 118.9 & 1.312 & 0.081 & 115.0 & 108.6 & 1.339 & 0.075 & 114.0 & 77.4 & 1.365 & 0.054 \\
\hline 118.0 & 82.2 & 1.322 & 0.055 & 116.0 & 78.9 & 1.342 & 0.054 & 115.0 & 60.9 & 1.365 & 0.042 \\
\hline 119.0 & 59.1 & 1.332 & 0.040 & 117.0 & 58.6 & 1.348 & 0.040 & 116.0 & 48.3 & 1.368 & 0.033 \\
\hline 120.0 & 44.2 & 1.342 & 0.029 & 118.0 & 44.7 & 1.354 & 0.030 & 117.0 & 38.9 & 1.371 & 0.026 \\
\hline 121.0 & 34.2 & 1.350 & 0.022 & 119.0 & 35.0 & 1.360 & 0.023 & 118.0 & 31.7 & 1.374 & 0.021 \\
\hline 122.0 & 27.2 & 1.356 & 0.018 & 120.0 & 28.1 & 1.365 & 0.019 & 119.0 & 26.3 & 1.377 & 0.018 \\
\hline
\end{tabular}

The absorption coefficient and refractive index of crystalline carbon dioxide ice derived from transmission measurements at 80,100 , and $120 \mathrm{~K}$ are given in Table I. Data are given for the spectral regions near the lattice bands. The absorption coefficient could not be obtained in the weakly absorbing inter-band region (where $\alpha_{p} \sim 0.001$ to $0.1 \mathrm{~cm}^{-1}$ ) using the thin film technique reported here. To obtain the absorption coefficient in these weakly absorbing regions, a technique for growing thick samples, such as the one developed by Hansen (1993), must be used.

In deriving the absorption coefficient for $\mathrm{CO}_{2}$ ice, a correction was made for instrumental drift in the reference transmission level $(<5 \%)$ over the course of the deposition and transmission measurements (typically 4-5 hr for $\mathrm{CO}_{2}$ ice). The drift in reference level appeared as a non-zero residual absorption in the weakly absorbing inter-band region of $\mathrm{CO}_{2}$ ice where the absorption coefficient is too small to be measured with this technique. A correction to the absorption coefficient baseline was made to remove a possible vertical shift in reference level and also for a change in the slope of the reference level over the spectral range. The function $f(v)=a_{0}+a_{1} v+a_{2} \cos \left(a_{3} v+a_{4}\right)$ was fitted to the baseline using a linear least-squares analysis and then subtracted from the measured absorption spec- 
trum. Residual interference fringes left by the inexact fringe fitting were also removed at the same time by fitting the amplitude, wavelength, and phase of the cosine term. Using this approach, the uncertainty in reference level could be reduced to below the instrumental noise. This approach, however, could not be applied to $\mathrm{H}_{2} \mathrm{O}$ ice measurements because water ice has measurable absorption over much of the far infrared spectral region.

The error in the derived absorption coefficient data arose from two main sources: systematic error in the film thickness and random error due to instrumental noise. Uncertainty in film thickness was due to inexact model fits to the interference fringes and, more significantly, to uncertainty in the real refractive index in this spectral region (see Appendix A for further discussion). The error in the $\mathrm{CO}_{2}$ absorption data due to the uncertainty in film thickness of approximately $3 \%$ was evaluated using the expression: $\sigma=\alpha_{\mathrm{p}} \cdot\left(\sigma_{d} / d\right)$, where $\alpha_{\mathrm{p}}$ is the absorption coefficient, $\sigma_{d}$ is the error in film thickness, and $d$ is the estimated film thickness. This yields an error in the derived absorption coefficient of approximately $\pm 6.5 \mathrm{~cm}^{-1}$ near the peaks of the lattice bands. The precision of the absorption coefficient was dominated by instrumental noise and was estimated to be $\pm 14.5 \mathrm{~cm}^{-1}$. The total uncertainty in the absorption coefficient data given in Table I considering these two error sources is less than $\pm 16 \mathrm{~cm}^{-1}$ near the lattice bands. Experimental error in the absorption coefficient propagates directly into the calculation of the imaginary refractive index and was evaluated using the expression $\sigma_{\mathrm{k}}^{2}=\left(\partial k / \partial \alpha_{\mathrm{p}}\right)^{2} \sigma_{\alpha_{\mathrm{p}}}^{2}=(1 / 4 \pi v)^{2} \sigma_{\alpha_{\mathrm{p}}}^{2}$, where $\sigma_{\mathrm{k}}$ and $\sigma_{\alpha_{\mathrm{p}}}$ are the errors in the imaginary refractive index and absorption coefficient, respectively. Evaluating this near the lattice bands gives errors of \pm 0.01 for the $112 \mathrm{~cm}^{-1}$ lattice band and \pm 0.02 for the $66.5 \mathrm{~cm}^{-1}$ lattice band.

The error in the real refractive indices given in Table I was dominated by uncertainty in the value of $n_{\infty}$ used in the Kramers-Kronig calculation, estimated to be \pm 0.05 . The precision in the real refractive index is on the order of \pm 0.01 due to uncertainty in the published near- and mid-infrared absorption coefficient data. The total error in the reported $n$ in Table $\mathrm{I}$ is \pm 0.06 .

The refractive index of $\mathrm{CO}_{2}$ ice derived from transmission measurements at $100 \mathrm{~K}$ is compared in Fig. 3 with data published by Warren (1986). The error bars represent the total uncertainty (systematic and random errors) in the imaginary refractive index and only the precision in the measured real refractive index reported in this study. The agreement is generally good with moderate disagreement in intensity and width of the $112 \mathrm{~cm}^{-1}$ lattice band. This lattice mode shows the greatest sensitivity to temperature. Warren extrapolated measurements made at several temperatures from 4.2 to $100 \mathrm{~K}$ by Kuan (1969). Only the spectral lineshape of the lattice bands measured at $4.2 \mathrm{~K}$ were plotted in Kuan (1969). Solid $\mathrm{CO}_{2}$ passes through a

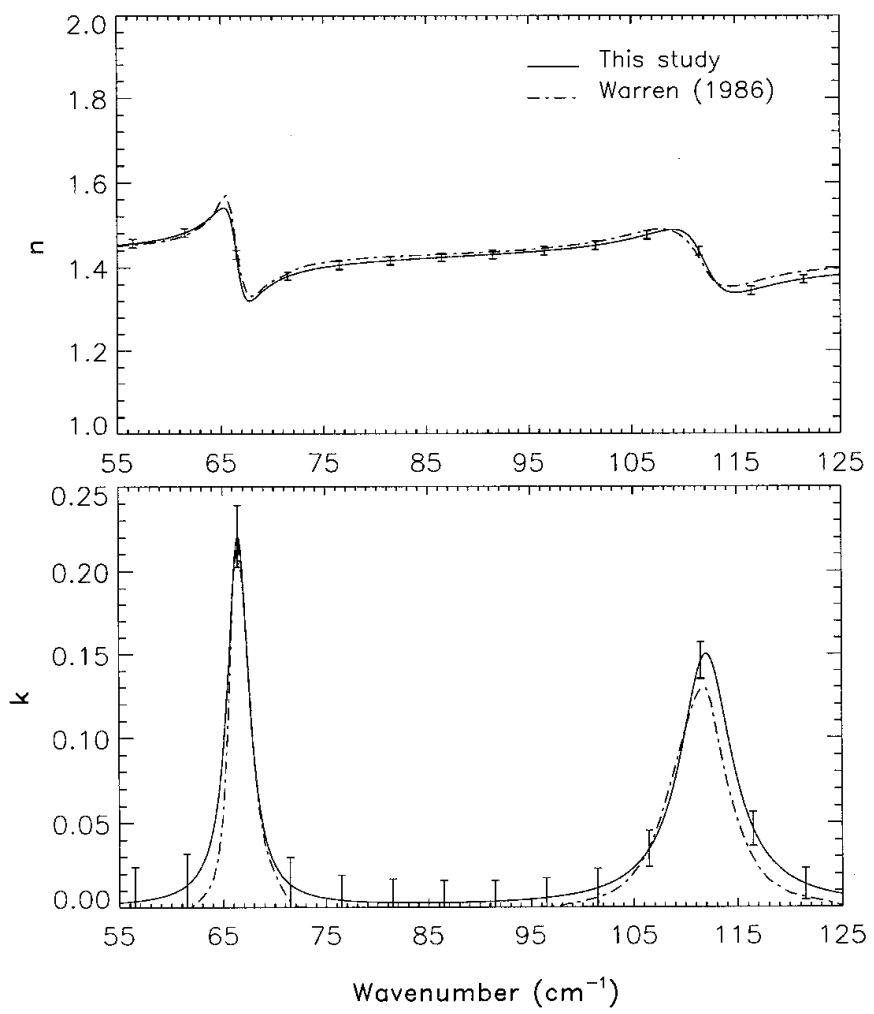

FIG. 3. Refractive index of $\mathrm{CO}_{2}$ ice at $100 \mathrm{~K}$ derived from measured transmission spectrum (solid lines). Top panel shows the real part of the refractive index, $n$, with the error bars representing the estimated precision. The bottom panel shows the imaginary part of the refractive index, $k$, with error bars representing the total uncertainty. Data published by Warren (1986) is shown for comparison (dashed-dotted line).

solid phase transition from amorphous to crystalline ice at temperatures around 30 to $50 \mathrm{~K}$, further complicating the extrapolation of lineshape. This extrapolation procedure may be partially responsible for the disagreement between the two sets of data near $112 \mathrm{~cm}^{-1}$. However, the consistently larger values of $k$ near $112 \mathrm{~cm}^{-1}$ suggests the 66 $\mathrm{cm}^{-1}$ lattice band may not have been fully resolved. The $1 \mathrm{~cm}^{-1}$ spectral resolution of the measurement could have underestimated the peak value by $\sim 10 \%$.

Table II contains the derived absorption coefficient and refractive indices of amorphous water ice at $77 \mathrm{~K}$ and crystalline water ice at 100 and $150 \mathrm{~K}$, respectively. The accuracy of the derived absorption coefficient for water ice and hence $k$ were mainly affected by a rather large uncertainty in film thickness of about $\pm 10 \%$ and the inability to adequately correct for reference level drift in the spectrometer. These two sources of uncertainty led to an error in absorption coefficient near the $230 \mathrm{~cm}^{-1}$ peak of approximately $\pm 325 \mathrm{~cm}^{-1}$. The precision in the water ice absorption coefficient was limited by instrumental noise to about $\pm 71 \mathrm{~cm}^{-1}$. The total uncertainty in the measured 
TABLE II

Measured Absorption Coefficients and Refractive Indices of Amorphous $\mathrm{H}_{2} \mathrm{O}$ Ice at $77 \mathrm{~K}$, and Crystalline (Ic) $\mathrm{H}_{2} \mathrm{O}$ Ice at 100 and $150 \mathrm{~K}$

$77 \mathrm{~K}$

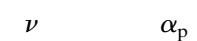

$\left(\mathrm{cm}^{-1}\right)$

$\left(\mathrm{cm}^{-1}\right)$

$n$

$k$

0.197

100.0

120.0

\section{0}

160.0

\section{0}

180.0

200.0

220.0

225.0

230.0

235.0

240.0

260.0

\section{0}

300.0

320.0

340.0

360.0

380.0

400.0

420.0

440.0

460.0

480.0

500.0

$\begin{array}{rl}238.8 & 1.842 \\ 549.5 & 1.846 \\ 734.6 & 1.683 \\ 887.0 & 1.597 \\ 867.2 & 1.548 \\ 943.9 & 1.548 \\ 1196.7 & 1.493 \\ 1491.4 & 1.427 \\ 1521.8 & 1.393 \\ 1686.5 & 1.227 \\ 1479.9 & 1.135 \\ 1274.9 & 1.138 \\ 822.9 & 1.131 \\ 561.3 & 1.149 \\ 374.4 & 1.190 \\ 218.0 & 1.219 \\ 67.8 & 1.282 \\ 12.8 & 1.346 \\ 3.3 & 1.401 \\ 78.0 & 1.411 \\ 177.1 & 1.434 \\ 290.8 & 1.447 \\ 435.6 & 1.447 \\ 527.9 & 1.435 \\ 722.1 & 1.422\end{array}$

0.364

0.418

0.441

0.418

0.417

$$
0.476
$$

0.539

0.538

0.584

0.501

0.423

0.252

0.160

0.099

0.054

0.016

0.003

0.001

0.016

0.034

0.053

0.075

0.088

0.115

$100 \mathrm{~K}$

$100 \mathrm{~K}$

\begin{tabular}{|c|c|c|c|c|c|c|c|}
\hline $\begin{array}{c}\nu \\
\left(\mathrm{cm}^{-1}\right)\end{array}$ & $\begin{array}{c}\alpha_{\mathrm{p}} \\
\left(\mathrm{cm}^{-1}\right)\end{array}$ & $n$ & $k$ & $\begin{array}{c}\nu \\
\left(\mathrm{cm}^{-1}\right)\end{array}$ & $\begin{array}{c}\alpha_{\mathrm{p}} \\
\left(\mathrm{cm}^{-1}\right)\end{array}$ & $n$ & $k$ \\
\hline 100.0 & 265.0 & 1.840 & 0.211 & 100.0 & 233.7 & 1.857 & 0.186 \\
\hline 120.0 & 535.6 & 1.799 & 0.355 & 120.0 & 577.6 & 1.800 & 0.383 \\
\hline 140.0 & 680.7 & 1.791 & 0.387 & 140.0 & 716.3 & 1.776 & 0.407 \\
\hline 160.0 & 1031.4 & 1.641 & 0.513 & 160.0 & 1113.5 & 1.589 & 0.554 \\
\hline 163.0 & 1088.6 & 1.576 & 0.531 & 160.5 & 1119.4 & 1.579 & 0.555 \\
\hline 180.0 & 729.0 & 1.584 & 0.322 & 180.0 & 795.5 & 1.605 & 0.352 \\
\hline 200.0 & 1121.5 & 1.614 & 0.446 & 200.0 & 1149.1 & 1.599 & 0.457 \\
\hline 220.0 & 1769.2 & 1.685 & 0.640 & 220.0 & 1906.5 & 1.641 & 0.690 \\
\hline 225.0 & 2211.7 & 1.730 & 0.782 & 215.0 & 1529.5 & 1.618 & 0.566 \\
\hline 230.5 & 3273.6 & 1.306 & 1.130 & 227.0 & 2717.6 & 1.360 & 0.953 \\
\hline 235.0 & 2496.9 & 0.989 & 0.846 & 235.0 & 1949.6 & 1.007 & 0.660 \\
\hline 240.0 & 1880.0 & 0.943 & 0.623 & 240.0 & 1686.2 & 1.032 & 0.559 \\
\hline 260.0 & 1044.6 & 1.043 & 0.320 & 260.0 & 1037.0 & 1.051 & 0.317 \\
\hline 280.0 & 673.7 & 1.045 & 0.191 & 280.0 & 629.7 & 1.071 & 0.179 \\
\hline 300.0 & 398.3 & 1.113 & 0.106 & 300.0 & 376.9 & 1.135 & 0.100 \\
\hline 320.0 & 187.1 & 1.168 & 0.047 & 320.0 & 200.7 & 1.186 & 0.050 \\
\hline 340.0 & 105.6 & 1.245 & 0.025 & 340.0 & 132.3 & 1.260 & 0.031 \\
\hline 360.0 & 79.2 & 1.299 & 0.017 & 360.0 & 122.2 & 1.315 & 0.027 \\
\hline 380.0 & 16.3 & 1.337 & 0.003 & 380.0 & 32.4 & 1.339 & 0.007 \\
\hline 400.0 & 40.1 & 1.348 & 0.008 & 400.0 & 62.3 & 1.353 & 0.012 \\
\hline 420.0 & 3.3 & 1.368 & 0.001 & 420.0 & 37.7 & 1.379 & 0.007 \\
\hline 440.0 & 0.0 & 1.390 & 0.000 & 440.0 & 28.3 & 1.397 & 0.005 \\
\hline 460.0 & 80.6 & 1.419 & 0.014 & 460.0 & 114.5 & 1.420 & 0.020 \\
\hline 480.0 & 209.1 & 1.425 & 0.035 & 480.0 & 277.9 & 1.430 & 0.046 \\
\hline 500.0 & 424.7 & 1.441 & 0.068 & 500.0 & 471.0 & 1.436 & 0.075 \\
\hline
\end{tabular}

$\mathrm{H}_{2} \mathrm{O}$ ice absorption coefficient data given in Table II is $\pm 333 \mathrm{~cm}^{-1}$, near the peak. This corresponds to an error in the imaginary refractive index of \pm 0.12 . The total uncertainty in the real refractive index of $\mathrm{H}_{2} \mathrm{O}$ ice was estimated to be \pm 0.06 .

The derived refractive index of water ice at $100 \mathrm{~K}$ is compared with data published by Bertie et al. (1969) in Fig. 4. Overall the agreement is fair with the most noticeable discrepancies in the peak and width of the $230 \mathrm{~cm}^{-1}$ feature. Some of this is likely due to the somewhat higher spectral resolution of $1-3 \mathrm{~cm}^{-1}$ used by Bertie et al. In our measurements, the spectral resolution was limited to $4 \mathrm{~cm}^{-1}$ by signal-to-noise considerations. In addition to this, Bertie et al. stated that by neglecting the reflection loss of approximately $27 \%$ in their analysis, they have likely overestimated the absorptivity in this region. We estimated this would result in an overestimate of approximately $10 \%$ in the measured peak absorption. The shoulder near $190 \mathrm{~cm}^{-1}$ reported by Bertie et al. was not observed in our measurements. Bertie et al. discuss the sources of experimental error in their measurements but a quantitative estimate of the error was not given. Further measurements with an improved technique for determining sample thickness are needed to resolve these discrepancies.

The cloud and surface ices on Mars form at higher temperatures than those measured in the laboratory. The surface temperatures in the polar regions are likely to be near $150 \mathrm{~K}$, the $\mathrm{CO}_{2}$ ice sublimation point at 6.1 mbars. The condensation temperatures for mid-latitude $\mathrm{H}_{2} \mathrm{O}$ ice cloud particles occur perhaps in the range 170 to $200 \mathrm{~K}$. These warmer ice temperatures cannot be achieved using thin film vacuum measurement techniques and therefore the refractive indices at higher temperatures were obtained by extrapolation.

The measured refractive indices of $\mathrm{CO}_{2}$ and $\mathrm{H}_{2} \mathrm{O}$ ices were extrapolated assuming a linear temperature dependence in integrated band strength, position, and width. This appears to be a very good assumption for $\mathrm{CO}_{2}$ ice (see Kuan 1969), but it is less clear how good the assumption is for $\mathrm{H}_{2} \mathrm{O}$ ice. Figure 5 shows the results of the extrapolation of refractive index of $\mathrm{CO}_{2}$ ice to $150 \mathrm{~K}$. Also shown for comparison are the measured refractive indices at 100 and $120 \mathrm{~K}$. Clearly, there is a significant change in the spectral characteristics, particularly in the band strength. $\mathrm{H}_{2} \mathrm{O}$ ice 


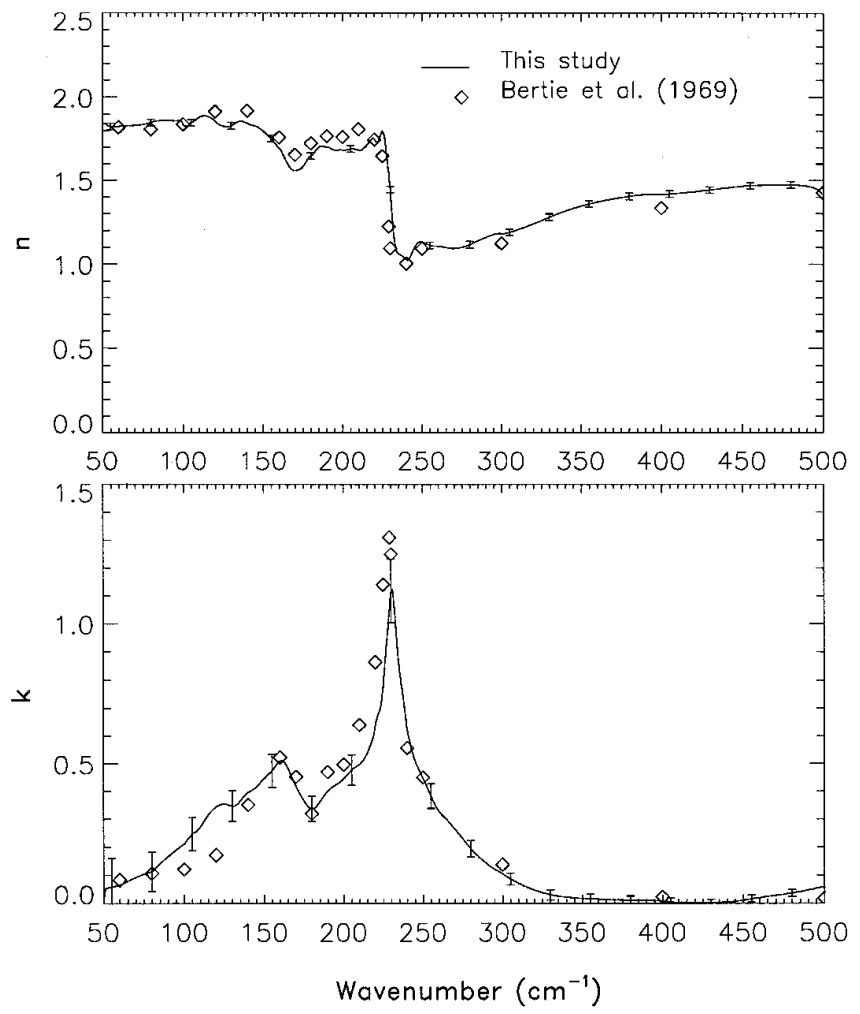

FIG. 4. Refractive index of $\mathrm{H}_{2} \mathrm{O}$ ice (Ic) at $100 \mathrm{~K}$ derived from measured transmission spectrum (solid lines). Top panel shows the real part of the refractive index, $n$, with error bars representing the estimated precision. The bottom panel shows the imaginary part of the refractive index, $k$, with error bars representing the total uncertainty. Data published by Bertie et al. (1969) is shown for comparison (diamonds).

refractive index was extrapolated to $200 \mathrm{~K}$ and the results are shown in Fig. 6 along with the refractive index measured at $100 \mathrm{~K}$. It must be pointed out that the extrapolated indices have rather large uncertainties associated with them. First, the linear temperature dependence may not be valid for such a large change in temperature, particularly as the ice approaches its sublimation temperature. Second, the uncertainties in the measured data used in the extrapolation are roughly on the same order of magnitude as changes with temperature. The error in the extrapolated values may be on the order of a factor of 2 to 3 . However, this was considered a necessary step in the modeling study until laboratory data become available for these warmer temperatures.

\subsection{Theoretical Emission Spectra}

Hapke's emittance model (Hapke 1993), and the laboratory data for $\mathrm{H}_{2} \mathrm{O}$ and $\mathrm{CO}_{2}$ ices were used to calculate theoretical far-infrared emissivities of martian polar ice caps. A brief description of Hapke's model is given in Appendix B. Hapke's model allows the spectral emissivity of a particulate surface to be calculated given the composition, particle sizes, packing density, and subsurface temperature gradient.

The sensitivity of calculated $\mathrm{CO}_{2}$ frost emissivity to particle size is shown in Fig. 7. Emissivities in this figure were calculated using the extrapolated refractive index for $\mathrm{CO}_{2}$ ice at $150 \mathrm{~K}$, assuming a packing density (ratio of the bulk density to particle density) of 0.9 and an isothermal subsurface temperature profile. Uncertainty in the imaginary refractive index can have a significant effect on the calculated emissivity. For example, in the weakly absorbing region (near $225 \mathrm{~cm}^{-1}$ ) with 1-mm-sized particles, the emissivity was calculated to be 0.8 for $k=10^{-3}$ and 0.5 for $k=10^{-4}$. The imaginary part of the $\mathrm{CO}_{2}$ ice refractive index was taken to be $10^{-4}$ in the weakly absorbing region (approximately 200 to $400 \mathrm{~cm}^{-1}$ ) for all the calculations used in this study. This value is consistent with values of absorption coefficient of less than $1 \mathrm{~cm}^{-1}$ in the range from 20 to $40 \mu \mathrm{m}$ measured by Hansen (1993). In addition, the influence of the far wing of the $660 \mathrm{~cm}^{-1} \mathrm{CO}_{2}$ ice absorption band and the $630 \mathrm{~cm}^{-1}$ isotopic band on the calculated frost emissivity at wavenumbers above $400 \mathrm{~cm}^{-1}$ was included using data from Warren (1986).

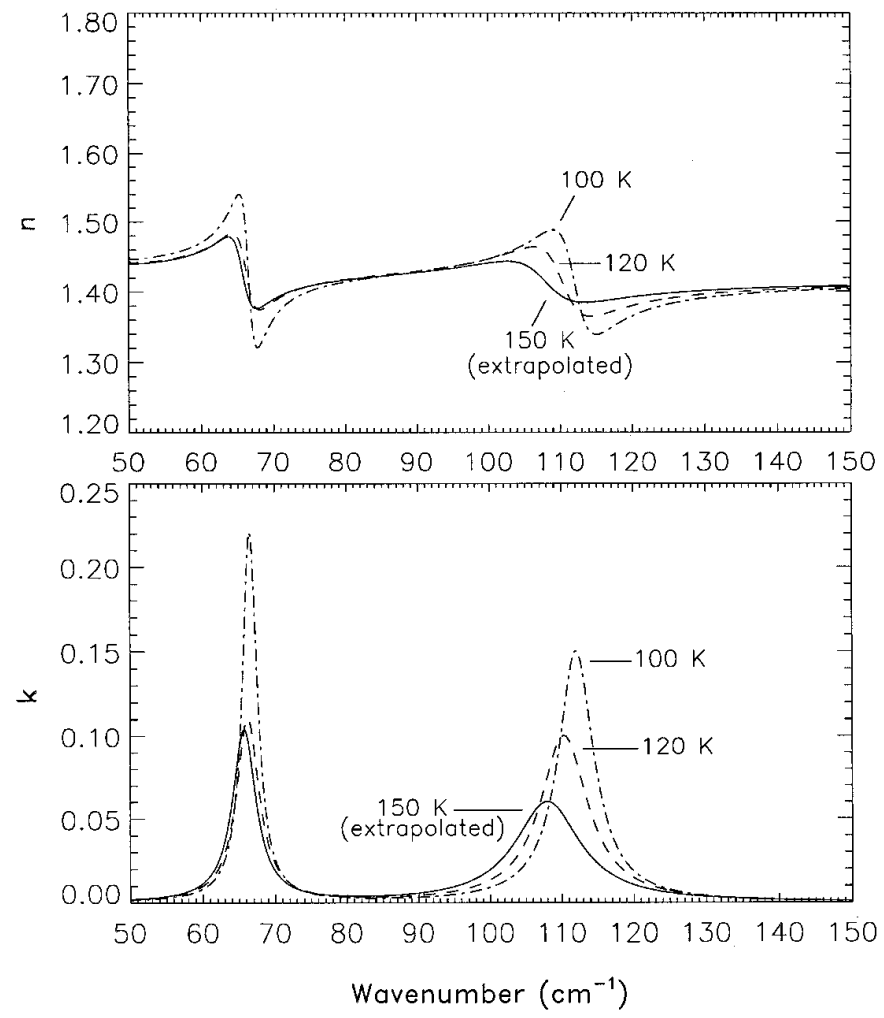

FIG. 5. Extrapolated $\mathrm{CO}_{2}$ ice refractive index at $150 \mathrm{~K}$ (solid line). Data extrapolated from measurements made at 80,100 , and $120 \mathrm{~K}$ assuming a linear temperature dependence in line position, integrated line strength, and width. Measured data at 100 and $120 \mathrm{~K}$ shown for comparison. 


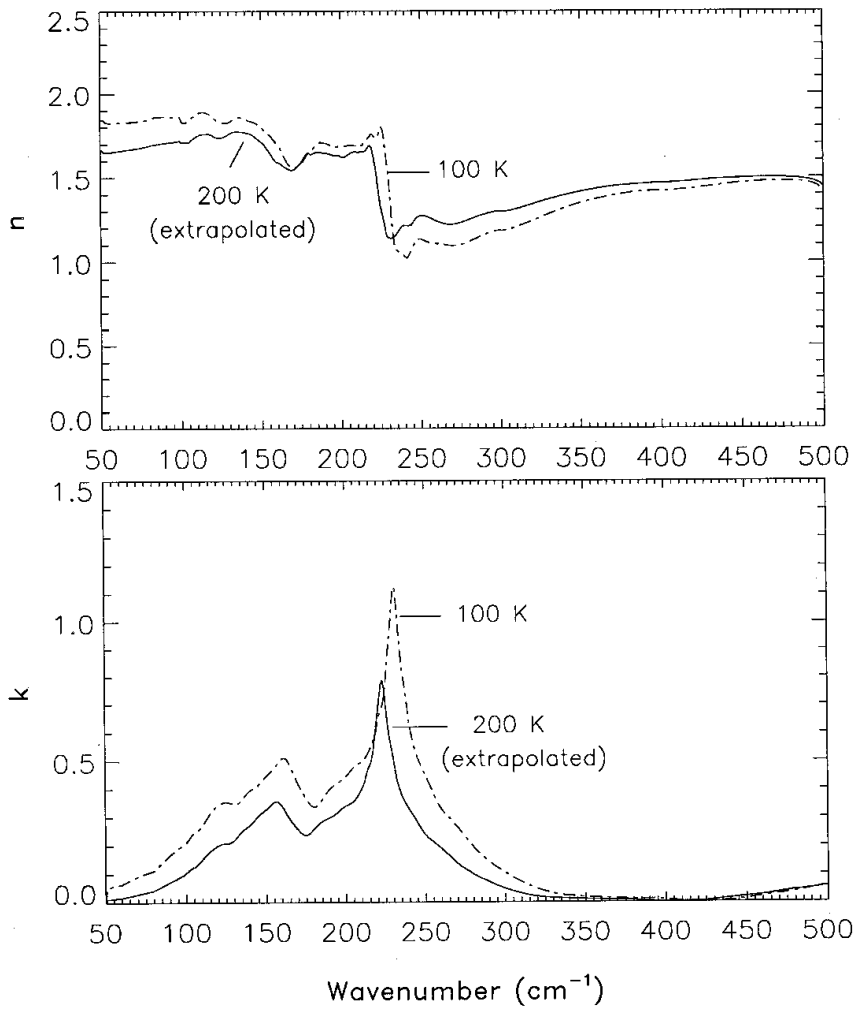

FIG. 6. Extrapolated $\mathrm{H}_{2} \mathrm{O}$ ice (Ic) refractive index at $200 \mathrm{~K}$ (solid line). Data extrapolated from measurements made at 100 and $150 \mathrm{~K}$, assuming a linear temperature dependence in line position, integrated line strength and width. Measured data at $100 \mathrm{~K}$ shown for comparison.

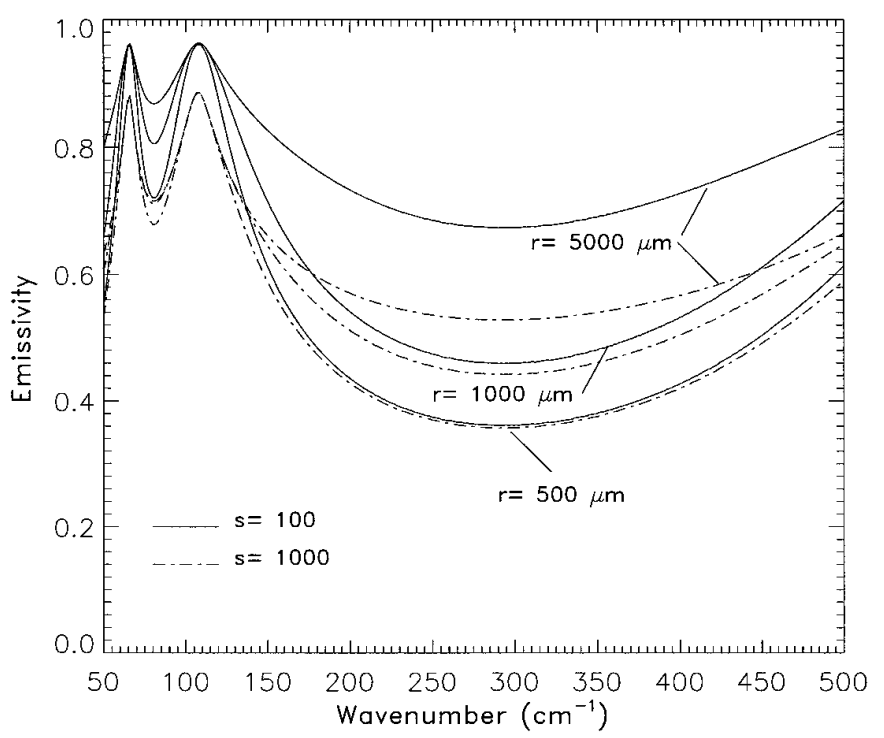

FIG. 7. Theoretical dependence of modeled $\mathrm{CO}_{2}$ frost emissivity on particle size and Hapke model parameter, $s$. Assumes a packing density of 0.9 and no subsurface temperature gradient.

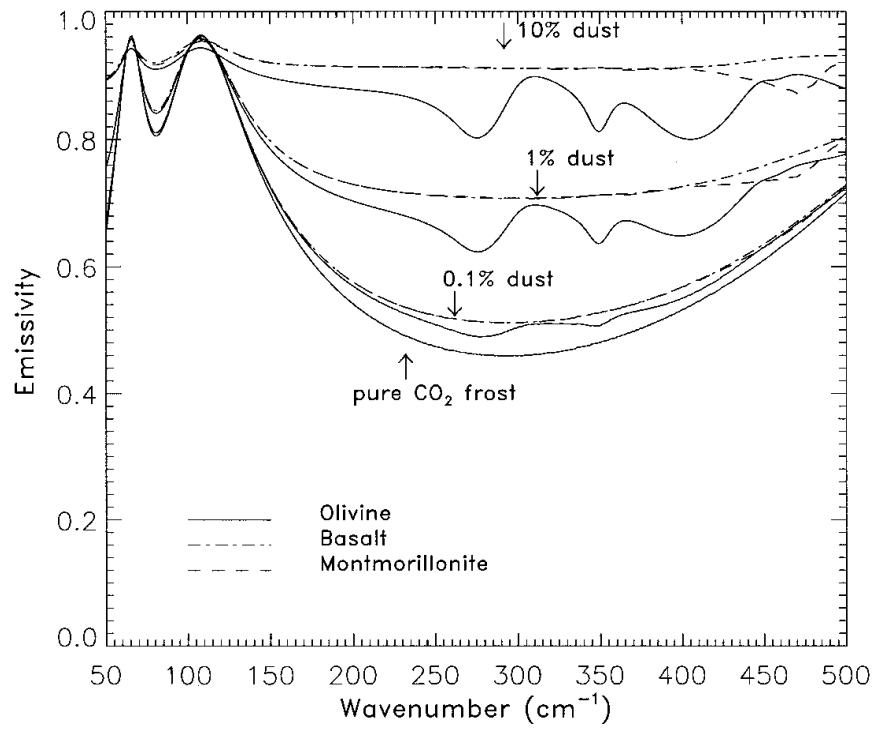

FIG. 8. Calculated emissivities of pure $\mathrm{CO}_{2}$ frost and $\mathrm{CO}_{2}$ frost mixed with various amounts of olivine particles (solid lines), basalt particles (dotted-dashed lines), and montmorillonite particles (dashed lines). See text for discussion of model parameters.

Hapke's model contains an adjustable parameter, $s$, which describes the internal volume scattering coefficient of the particle. Two sets of curves are shown in Fig. 7, one corresponding to $s=100$ (solid lines) and another corresponding to $s=1000$ (dotted-dashed lines). A reasonable value for $s$ based on physical arguments is very difficult to make. The value of $s$ has a significant effect on the spectral characteristics of the frost. Increasing $s$ results in greater internal scattering of photons, increasing the absorption path length and ultimately decreasing the number of photons escaping the surface. This is seen in Fig. 7 as an overall decrease in frost emissivity with an increased value of $s$. The decrease in $\mathrm{CO}_{2}$ ice grain size also leads to a decrease in frost emissivity due to the greater number of grain interfaces and the correspondingly greater number of surface reflections at these interfaces, again increasing the absorption path length. This spectral dependence on particle size is consistent with the type III spectral behavior for particulate surfaces discussed by Hunt and Vincent (1968) and more recently by Moersch and Christensen (1995). The lattice band shapes appear to be less dependent on grain size as the volume scattering coefficient increases. The sensitivity of far-infrared $\mathrm{CO}_{2}$ frost emissivity to grain sizes and refractive index is qualitatively similar to the behavior seen in mid-infrared emissivities modeled by Warren et al. (1990).

The modeled $\mathrm{CO}_{2}$ frost emissivity is sensitive to the presence of dust or $\mathrm{H}_{2} \mathrm{O}$ ice particles in the frost. Figure 8 shows the dependence of frost emissivity on various amounts of dust. In this figure, the $\mathrm{CO}_{2}$ ice particle sizes 


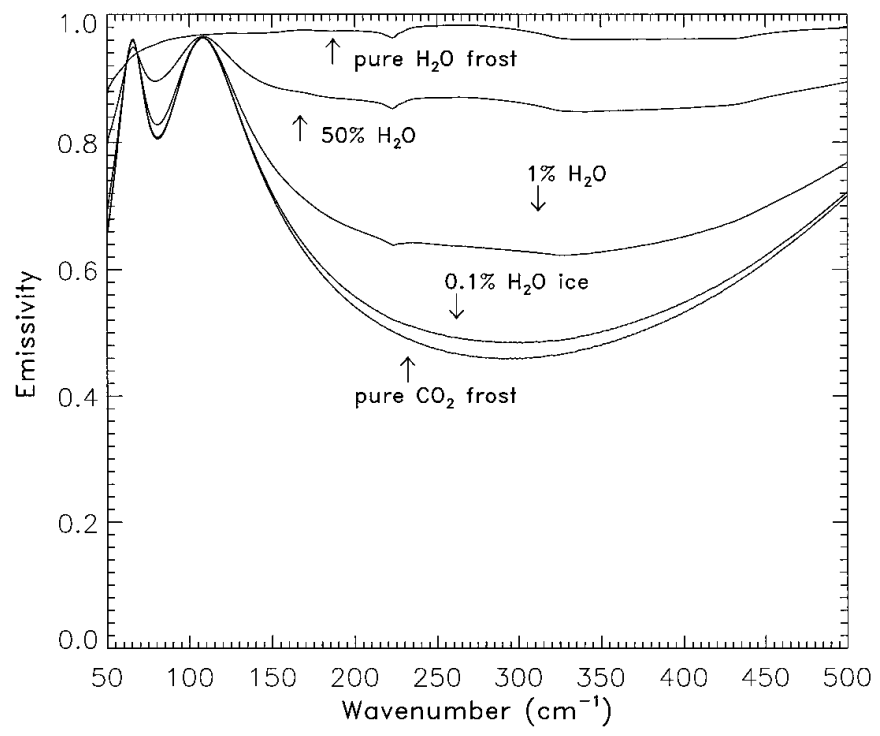

FIG. 9. Calculated emissivities of pure $\mathrm{CO}_{2}$ and $\mathrm{H}_{2} \mathrm{O}$ frosts, and frost mixed with various amounts of $\mathrm{CO}_{2}$ and $\mathrm{H}_{2} \mathrm{O}$ particles. See text for discussion of model parameters.

are $1 \mathrm{~mm}$ with dust particle sizes of $5 \mathrm{~mm}$, and both having a volume scattering coefficient equal to 100 . The refractive indices of olivine, basalt (Pollack et al. 1973), and montmorillonite (Toon et al. 1977) were used to model the spectral properties of the dust. The refractive indices for these materials were extended below $200 \mathrm{~cm}^{-1}$ for basalt and $250 \mathrm{~cm}^{-1}$ for montmorillonite $(219 \mathrm{~b})$ by assuming that the imaginary refractive index for these materials decreases smoothly with decreasing wavenumber to a value of 0.02 at $50 \mathrm{~cm}^{-1}$. This follows the general spectral behavior of olivine at the longer wavelengths.

All three materials produce approximately the same increase in frost emissivity with increasing amounts of dust in the weakly absorbing inter-band region of $\mathrm{CO}_{2}$ ice. Olivine exhibits the greatest spectral variation in the mixed frost emissivity. The lattice band emissivities show a small decrease with increasing amounts of olivine dust, but very little change for basalt and montmorillonite. The actual change in emissivity due to the presence of dust is dependent on the assumed particle sizes of the dust with smaller dust grains showing less influence on the emissivity. The dust grain sizes considered here are much larger than what would likely be present in the martian polar ice caps, but the large particle limitations of Hapke's model precludes an adequate study of the effects of smaller grain sizes.

The presence of $\mathrm{H}_{2} \mathrm{O}$ ice particles in $\mathrm{CO}_{2}$ frost also affects the modeled emissivity. The emissivity of $\mathrm{CO}_{2}$ frost mixed with various amounts of $\mathrm{H}_{2} \mathrm{O}$ ice particles ranging from 0.1 to $50 \%$ are shown in Fig. 9. The $\mathrm{H}_{2} \mathrm{O}$ ice particles have a radius of $1 \mathrm{~mm}$ and the model parameters for $\mathrm{CO}_{2}$ frost are the same as described above. Pure $\mathrm{H}_{2} \mathrm{O}$ frost emissivity is given by the top curve in Fig. 9. $\mathrm{H}_{2} \mathrm{O}$ frost has emissivity close to unity throughout most of the farinfrared, showing very little spectral variation. $\mathrm{H}_{2} \mathrm{O}$ frost emissivity is insensitive to particle sizes in the range from $500 \mu \mathrm{m}$ to $5 \mathrm{~mm}$. The sensitivity of $\mathrm{H}_{2} \mathrm{O}$ and $\mathrm{CO}_{2}$ frost emissivities to moderate subsurface temperature gradients $(0.01$ to $1.0 \mathrm{~K} / \mathrm{cm})$ and packing density was found to be relatively small.

Two conclusions relevant to the far-infrared detection of $\mathrm{H}_{2} \mathrm{O}$ and $\mathrm{CO}_{2}$ frosts emerge from the modeling study. The calculations show that there is very little spectral variation in the far-infrared emissivity of pure $\mathrm{H}_{2} \mathrm{O}$ frost composed of large particles. This suggests that detection of $\mathrm{H}_{2} \mathrm{O}$ frost based on its unique far-infrared spectral signature will be extremely difficult. For $\mathrm{CO}_{2}$ frost, however, the strong spectral variation between its lattice band emissivities and the nearby weakly absorbing inter-band region is quite significant. This large change in emissivity gives rise to a large change in the spectral brightness temperature of $\mathrm{CO}_{2}$ frost. This spectral behavior has important implications for remotely detecting the presence of $\mathrm{CO}_{2}$ frosts and possibly other solar system ices, such as $\mathrm{N}_{2}$ and $\mathrm{CH}_{4}$, that possess infrared-active lattice absorption bands and also appear to exhibit weak inter-band absorption in the far-infrared. In addition, the emissivity of $\mathrm{CO}_{2}$ frost is observably affected by the presence of small amounts $(0.1$ to $1.0 \%)$ of dust or $\mathrm{H}_{2} \mathrm{O}$ contaminants in the frost. In principle, therefore, the spectral region between about 200 and $500 \mathrm{~cm}^{-1}$ could be used to detect the presence of small amounts of contaminants in the Martian polar $\mathrm{CO}_{2}$ ice caps. Unfortunately, the emissivity in this spectral region is also quite sensitive to particle sizes, uncertainty in the imaginary refractive index, and possible uncertainty in the values of model parameters, such as the internal volume scattering coefficient of the particles. This will likely lead to ambiguity in interpreting frost observations in this spectral region.

The spectral characteristics of mid-latitude water ice clouds were studied by incorporating the laboratory data for water ice extrapolated to $200 \mathrm{~K}$ into a detailed radiative transfer model (see Appendix B). Calculations using the mid-latitude cloud models based on Mariner 9, Viking and Phobos spacecraft observations were made. It was found that except for cloud models with total water ice column amounts greater than 1 to $2 \mathrm{pr} \mu \mathrm{m}$, the far-infrared spectral emission did not show observable water ice cloud features against the relatively warm (e.g., $220 \mathrm{~K}$ ) surface background radiance. For example, the cloud models based on Kahn's analysis of Viking images having vertical integrated cloud ice column amounts $\leq 0.01 \mathrm{pr} \mu \mathrm{m}$ and on the range of haze parameters derived from Phobos observations were too optically thin in the far infrared $\left(\tau \leq 10^{-4}\right)$ to be detectable. Even the optically thicker cloud model corresponding to Mariner 9 observations in the Tharsis Ridge region, with a cloud ice amount of $0.4 \mathrm{pr} \mu \mathrm{m}$ and a corresponding far- 
infrared optical depth of 0.05 , did not show a clear cloud spectral feature in the calculated emission spectrum for the nadir viewing geometry assumed in this study. An observable cloud feature was produced in the calculated spectrum using a simple cloud model with an integrated ice amount of $2 \mathrm{pr} \mu \mathrm{m}$, a condensation temperature of about $200 \mathrm{~K}$, and a surface temperature of $225 \mathrm{~K}$. The resulting far infrared optical depth was $\sim 0.16$. The 225 $\mathrm{cm}^{-1}$ water ice absorption band was observed in the theoretical spectrum by differencing the cloud spectrum with a cloud-free spectrum resulting in a brightness temperature difference of $\Delta T=2.5 \mathrm{~K}$. The weaker ice absorption near $158 \mathrm{~K}$ was only marginally observable in the difference spectrum. The cloud ice amount in this case was probably near the upper limit for the martian atmosphere based on arguments made by Rossow (1978), who used a simple theoretical cloud microphysical model for martian water ice clouds.

The far infrared spectra of martian water ice clouds were found to be most sensitive to total cloud ice amount and to the physical temperature difference between the cloud and the underlying surface. The theoretical spectra were insensitive to moderate amounts of atmospheric dust (visible optical depth $<3$ ) and gaseous absorption under typical mid-latitude conditions. The effect of atmospheric dust opacity is to modify the apparent surface emissivity, changing the cloud-surface effective radiating temperature difference. An approximate radiative transfer model was developed to investigate the sensitivity of water ice cloud spectra to a range of cloud ice amounts and cloud-surface temperature differences. The model assumes an isothermal cloud having a uniform optical depth, and a gray emitting surface. To a first approximation, the far infrared optical depth is independent of particle size (for $r_{\text {eff }}<6 \mu \mathrm{m}$ ) and depends directly on column ice amount, given in terms of precipitable micrometers. This can be shown using the following relationship for optical depth: $\tau=N_{\mathrm{col}} \cdot \sigma_{\mathrm{ext}}$, with the column number density given by $N_{\mathrm{col}}=\left(\rho_{\mathrm{w}} / \rho_{\mathrm{i}}\right) \cdot\left(\Delta_{\mathrm{pr} \mu \mathrm{m}} /\right.$ $\left.\frac{4}{3} \pi r_{\mathrm{e}}^{3}\right)$, where $\rho_{\mathrm{w}}$ and $\rho_{\mathrm{i}}$ are the densities of water and ice, respectively, $\Delta_{\mathrm{pr} \mu \mathrm{m}}$ is the column amount in precipitable micrometers, and $r_{\mathrm{e}}$ is the effective radius of the cloud particle. The extinction cross section for water ice particles at $225 \mathrm{~cm}^{-1}$ is $\sigma_{\text {ext }}=\left(r_{\mathrm{e}} / 0.15\right)^{3} \cdot 9 \times 10^{-12} \mathrm{~cm}^{2}$, assuming a modified gamma size distribution with $\alpha=\gamma=1$.

The results of calculations using this simplified model of mid-latitude water ice clouds for a range of cloud ice amounts, and cloud and surface temperatures are shown in Fig. 10, assuming a surface emissivity of 0.93 . The spectral brightness temperature contrast $\left(\Delta T_{\mathrm{b}}\right)$ is defined as the difference between the calculated brightness temperature at the peak of the water ice spectral feature (i.e., $225 \mathrm{~cm}^{-1}$ ) and the surface brightness temperature where the cloud opacity is zero. The cloud and surface temperatures are given in terms of a difference between the ground $\left(T_{\mathrm{g}}\right)$

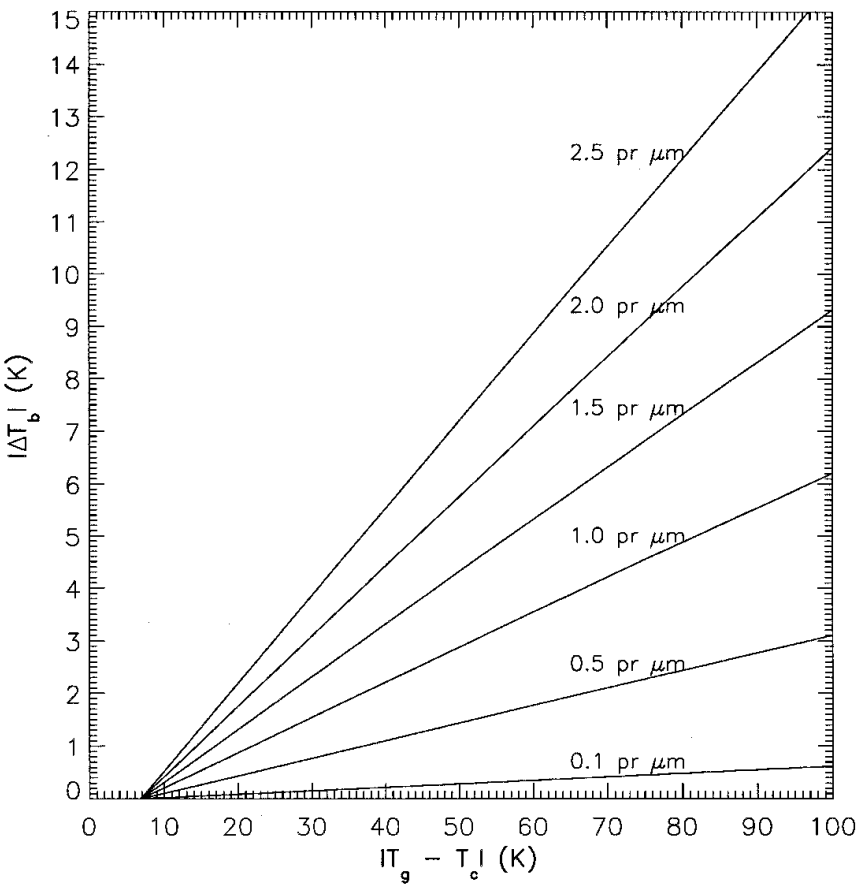

FIG. 10. Dependence of spectral brightness temperature contrast $\left(\left|\Delta T_{\mathrm{b}}\right|\right)$ of modeled mid-latitude $\mathrm{H}_{2} \mathrm{O}$ ice clouds on the difference between ground temperature $\left(T_{\mathrm{g}}\right)$ and cloud temperature $\left(T_{\mathrm{c}}\right)$ for various cloud ice amounts. The spectral brightness temperature contrast is defined as the difference between the brightness temperature observed at the peak of the cloud spectral feature (e.g., $225 \mathrm{~cm}^{-1}$ for water ice) and the nearby continuum brightness temperature where the cloud optical depth is zero. In the typical mid-latitude case where a cold cloud is observed against a relatively warm surface, the spectral feature is seen in absorption (i.e., $\left.\Delta T_{\mathrm{b}}<0\right)$.

and cloud $\left(T_{\mathrm{c}}\right)$ temperatures. Note that for typical midlatitude conditions, the cloud is colder than the underlying surface and the cloud ice spectral feature will be seen in absorption (i.e., $\Delta T_{\mathrm{b}}<0$ ). Cloud models based on Viking and Phobos observations fall well below the $0.1 \mathrm{pr} \mu \mathrm{m}$ curve in Fig. 10. The Tharsis Ridge cloud model would fall just below the $0.5 \mathrm{pr} \mu \mathrm{m}$ curve. From Kahn's analysis, it appears that there is a tendency for low clouds $(<20$ $\mathrm{km})$ to have a lower cloud condensation temperature and a greater cloud ice amount than high clouds $(>50 \mathrm{~km})$, where the opposite is true. This tendency reduces the spectral brightness contrast to very small values $\left(\Delta T_{\mathrm{b}}<0.5\right)$ for a large range of mid-latitude cloud conditions. Water ice clouds forming near the edge of the retreating seasonal ice caps during springtime have a significantly greater cloud ice amount approaching 1.5 to $2.0 \mathrm{pr} \mu \mathrm{m}$. This would lead to values of $\Delta T_{\mathrm{b}} \sim 2$ to $3 \mathrm{~K}$.

The effects of suspended dust on the observed surface emissivity was also investigated using the simplified radiative transfer model. In Fig. 11, the apparent change in observed surface emissivity at 200 to $250 \mathrm{~cm}^{-1}$ due to a 


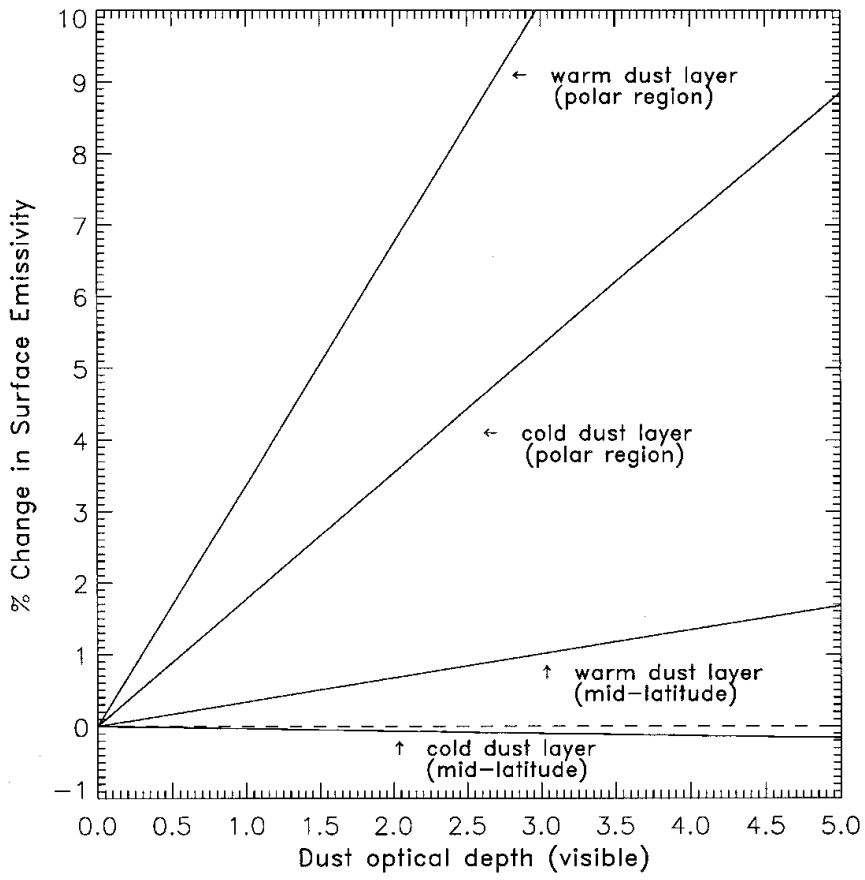

FIG. 11. Apparent change in observed surface emissivity at $225 \mathrm{~cm}^{-1}$ due to an overlying layer of suspended dust. Dust amount is given in terms of its visible optical depth. For this calculation, the surface emissivity was assumed to be 0.44 ( $\mathrm{CO}_{2}$ frost) for the polar region and 0.93 (regolith) for the mid-latitude region. A warm dust layer was taken to be $10 \mathrm{~K}$ warmer than the underlying surface, while a cold dust layer was taken to be $10 \mathrm{~K}$ colder than the surface.

layer of suspended dust is shown as the difference between the actual surface emissivity and the apparent or effective emissivity. Dust amounts are given in terms of visible optical depth. For this calculation, the surface emissivity was assumed to be $0.44\left(\mathrm{CO}_{2}\right.$ frost $)$ for the polar region and 0.93 (regolith) for the mid-latitude region. A warm dust layer was taken to be $10 \mathrm{~K}$ warmer than the underlying surface, while a cold dust layer was taken to be $10 \mathrm{~K}$ colder than the surface. The presence of a dust layer would have little affect on observed mid-latitude surface brightness temperature in the far infrared, even for situations where large dust loading occurs. The influence of atmospheric dust on the observed brightness temperature of the seasonal polar ice caps would be on the order of a few percent. In this case, observations of the polar $\mathrm{CO}_{2}$ ice caps may not be capable of distinguishing between the effects of atmospheric dust and dust incorporated into the polar caps.

In addition to the mid-latitude calculations, the far-infrared spectra of polar condensates and surface ices were calculated using the detailed radiative transfer model. The result of the radiative transfer calculation for a polar springtime water ice cloud and $\mathrm{CO}_{2}$ surface ice model, a situation typical near the polar cap edge, is shown in Fig. 12. In this model, the water ice cloud was located at $15-\mathrm{km}$ altitude having a modified gamma particle size distribution with mode radius equal to $0.5 \mu \mathrm{m}, \alpha=\gamma=1$, and a total ice column amount of $2 \mathrm{pr} \mu \mathrm{m}$. Thermal emission from a pure $\mathrm{CO}_{2}$ frost (dotted-dashed line) shows two observable emission features at 66 and $110 \mathrm{~cm}^{-1}$, due to the lattice absorption bands in solid $\mathrm{CO}_{2}$. At larger wavenumbers, the frost emissivity is very low, resulting in brightness temperatures far below the $150 \mathrm{~K}$ physical temperature of the modeled frost. The pure $\mathrm{CO}_{2}$ frost emission curve neglects absorption or emission by the atmosphere. The solid line curve in Fig. 12 is the combined atmosphere and surface emission. Several narrow water vapor lines are seen in emission against the cold surface background. The atmosphere was assumed to be free of suspended dust in this case. A water ice cloud emission feature is clearly seen at $225 \mathrm{~cm}^{-1}$ with the low surface emissivity of $\mathrm{CO}_{2}$ frost providing greater spectral contrast of the cloud feature than was seen in the mid-latitude calculations. Also shown in Fig. 12 is the emission spectrum for the same cloud and surface models, but with a moderately dusty atmosphere, having a visible dust optical depth equal to 1.0 (dotted line). In this last case, the water ice cloud feature is still observable near $225 \mathrm{~cm}^{-1}$ and a second spectral feature appears near $290 \mathrm{~cm}^{-1}$ due to emission by olivine dust. This atmospheric dust feature can be readily distinguished from the surface material because it is seen in emission instead of absorption (see Fig. 8 for comparison).

The far infrared spectrum of the martian polar hood is shown in Fig. 13. The polar hood aerosol model consisted of a low-lying $\mathrm{CO}_{2}$ ice haze, a mid-level $\mathrm{H}_{2} \mathrm{O}$ ice cloud, and atmospheric dust particles. The $\mathrm{CO}_{2}$ ice haze was modeled as a near-surface diffuse layer approximately $3 \mathrm{~km}$ deep with an integrated cloud ice amount of $\sim 10 \mathrm{pr} \mu \mathrm{m}$. The $\mathrm{CO}_{2}$ ice particle sizes were assumed to follow a modified gamma distribution with an effective radius of $10 \mu \mathrm{m}$ and $\alpha=\gamma=1$, resulting in a visible optical depth of 2.0. The polar $\mathrm{H}_{2} \mathrm{O}$ ice cloud model was similar to the previous polar springtime cloud model example, but with a lower integrated cloud amount of $1 \mathrm{pr} \mu \mathrm{m}$. The water ice cloud visible optical depth was 0.65 . Even with this lower cloud density the water ice feature is still observable in the calculated spectrum. The atmospheric dust amount in the polar hood model corresponded to "clear air" conditions with a visible opacity of 0.5 . The standard dust model parameters were assumed. The pure $\mathrm{CO}_{2}$ frost emission curve, neglecting atmospheric effects, is shown for comparison (dotteddashed line). The $\mathrm{CO}_{2}$ frost emission features at 66 and $110 \mathrm{~cm}^{-1}$ are clearly visible. Also present in the spectrum is a weak emission feature near $290 \mathrm{~cm}^{-1}$ from the atmospheric olivine dust layer.

Scattering by the $\mathrm{CO}_{2}$ haze layer lowers the apparent surface brightness temperature below that expected for a pure frost. Over much of the far-infrared region, the single scattering albedo for $\mathrm{CO}_{2}$ ice particles is close to one, 


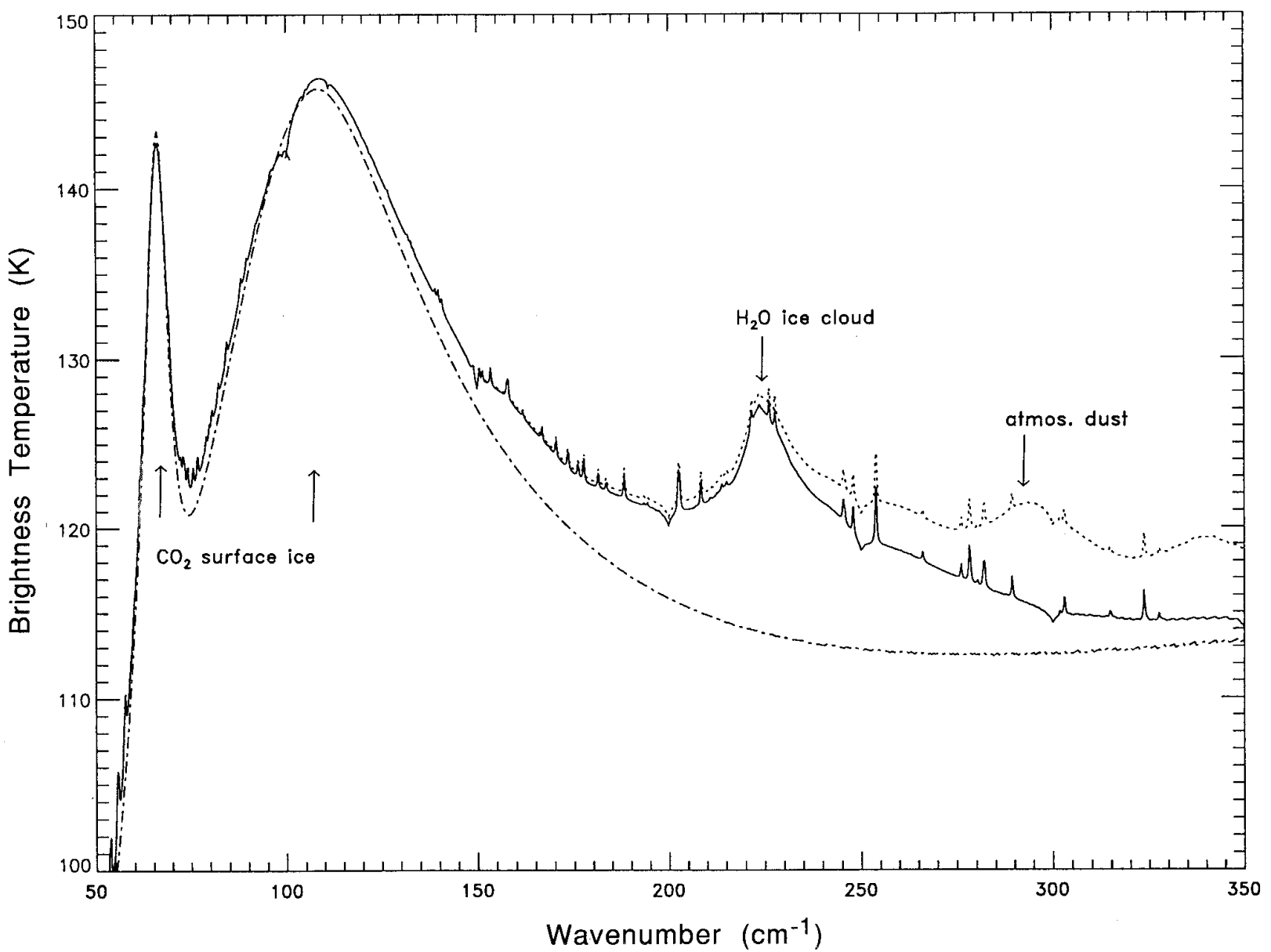

FIG. 12. Theoretical far-infrared emission spectrum of a typical polar springtime $\mathrm{H}_{2} \mathrm{O}$ ice cloud model with an ice column amount of 2 pr $\mu \mathrm{m}$ (solid line). The same emission spectrum including suspended dust having a visible optical depth of 1.0 is also shown (dotted line). The brightness temperature of a pure $\mathrm{CO}_{2}$ surface frost at a physical temperature of $150 \mathrm{~K}$ is shown for comparison (dashed-dotted line).

whereas for water ice and dust it is close to zero. The high scattering albedo of $\mathrm{CO}_{2}$ ice particles results in a very low emissivity haze. This is consistent with the results obtained by Hunt (1980), who was able to show that a $\mathrm{CO}_{2}$ ice cloud with a visible optical depth of 0.5 can have a large effect on the measured $20-\mu \mathrm{m}$ brightness temperatures in the polar regions. The measured brightness temperature can be as low as 120 to $130 \mathrm{~K}$ for a physical surface temperature of $150 \mathrm{~K}$. This effect could be used to detect the presence of a low-lying $\mathrm{CO}_{2}$ ice haze, but quantitative measurements would be difficult due to uncertainties in modeling the underlying frost emissivity.

\section{FEASIBILITY OF DETECTION}

A preliminary assessment of the observational requirements for an earth-based instrument was made based on the results of the laboratory measurements and the theoretical modeling studies. Three fundamental requirements emerged from this study: a need for high spatial resolution, high instrument sensitivity, and a high-altitude platform to make observations from. Spectral resolution is not a critical requirement since resolution on the order of $1 \mathrm{~cm}^{-1}$ is sufficient and this can be easily accomplished using a grating spectrometer or a Fourier transform spectrometer.

To achieve adequate spatial resolution to resolve the limited horizontal extent of the polar caps on Mars or the small angular extent of the moons in the outer solar system with a single telescope requires a large primary mirror. A large mirror will also increase the amount of light-gathering power of a telescope, improving the sensitivity. A telescope operating at the diffraction limit will have a full angular field of view in arcseconds given by $\alpha=0.412 \lambda / D$, where $\lambda$ is the operating wavelength in $\mu \mathrm{m}$ and $D$ is the diameter of the primary mirror in meters. In the case of Mars, a 4$\mathrm{m}$-diameter mirror is required to just match the angular field of view of the telescope to the angular extent of Mars (taken to be $20 \operatorname{arcsec}$ in this analysis) at $50 \mathrm{~cm}^{-1}$ and a 


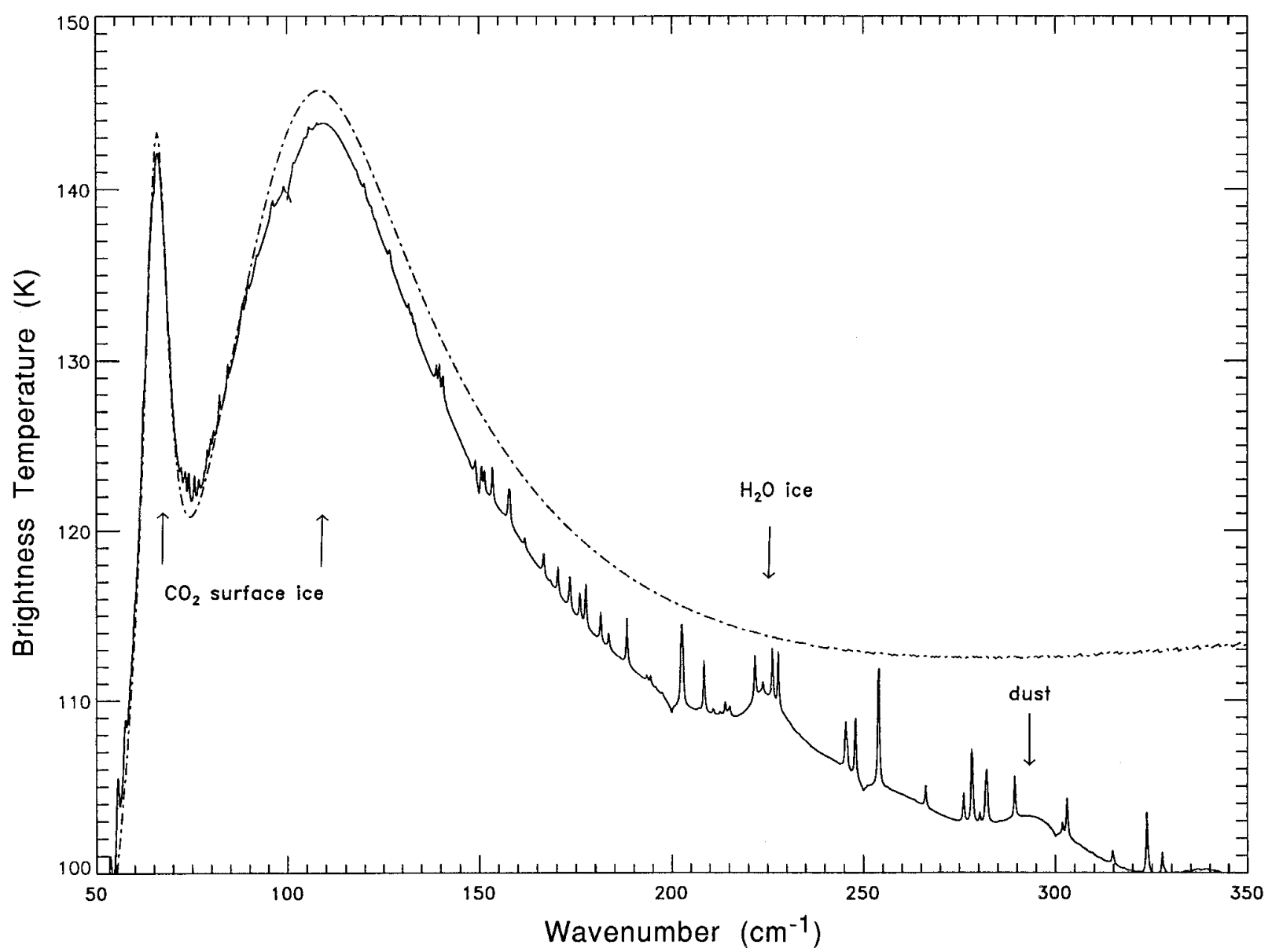

FIG. 13. Theoretical far infrared spectrum of polar hazes consisting of atmospheric $\mathrm{CO}_{2}$ ice particles with an integrated column ice amount of $10 \mathrm{pr} \mu \mathrm{m}, \mathrm{H}_{2} \mathrm{O}$ ice particles with an integrated column ice amount of $1 \mathrm{pr} \mu \mathrm{m}$, and suspended dust particles with a visible optical depth of 0.5 (solid line). The brightness temperature of a pure $\mathrm{CO}_{2}$ surface frost at a physical temperature of $150 \mathrm{~K}$ is shown for comparison (dashed-dotted line).

2-m mirror is needed at $100 \mathrm{~cm}^{-1}$. A mirror diameter of $14 \mathrm{~m}$ operating at $50 \mathrm{~cm}^{-1}$ is required to resolve the polar region on Mars (roughly 6 arcsec). Large ground-based observing telescopes are feasible but the far-infrared opacity of the earth's atmosphere, primarily from water vapor, precludes observations from the ground (Taub and Stier 1976).

Observations made at aircraft or balloon altitudes would be above much of the atmospheric water vapor. However, these platforms place practical constraints on the mirror size, limiting it to 1 or $2 \mathrm{~m}$. An earth-orbiting satellite, such as the Large Deployable Reflector (LDR) satellite for submillimeter observations with a primary mirror diameter between 10 and $20 \mathrm{~m}$ (Swanson, 1991) would provide an ideal platform for observing martian surface ices. An alternate approach must be considered for observations of icy moons in the outer solar system. Their very small angular sizes as seen from the earth make the spatial resolution requirements of a far-infrared observation significantly more stringent. For instance, the angular sizes of Titan and Triton are $\sim 1$ arcsec and $\sim 0.13$ arcsec, respectively. In addition, sufficiently high off-axis rejection must be achieved to isolate the flux from the moon and its nearby planet. For example, in the Neptune/Triton system, the angular separation, as seen from the earth, between Neptune and Triton is approximately $30 \mathrm{arcsec}$. Interferometric techniques involving two or more telescopes on a single satellite or on multiple satellites are theoretically capable of achieving very high angular resolution of the order of tenths of arcseconds.

Another important consideration in evaluating the feasibility of detecting planetary ices is the sensitivity required to measure weak spectral features. The signal-to-noise ratio for a direct detection system is given by $S / N=\eta S_{0} A_{\mathrm{m}}$ $\Delta \nu \Omega_{\mathrm{s}} \sqrt{t} / N E P$, where $\eta$ is the instrument transmission, $S_{\mathrm{o}}$ is the source flux, $A_{\mathrm{m}}$ is the collecting area of the primary mirror, $\Delta v$ is the spectral resolution, $\Omega_{\mathrm{s}}$ is the solid angle of the source, $t$ is the integration time, and NEP is the 
noise equivalent power of the detector. The predicted sensitivity of future $\mathrm{Si}$ or $\mathrm{Ge}$ bolometers cooled to liquid helium temperatures is about $5 \times 10^{-19} \mathrm{~W} / \mathrm{Hz}^{1 / 2}$ (McCreight 1991). Evaluating the expression for the signalto-noise ratio for observation of the martian polar ice caps assuming an operating frequency of $100 \mathrm{~cm}^{-1}$, a $1 \mathrm{~cm}^{-1}$ spectral resolution, a $10-\mathrm{m}$ mirror, $\eta=0.3, S_{\mathrm{o}}=1.131 \times$ $10^{-10} \mathrm{~W} / \mathrm{m}^{2} \mathrm{sr} \mathrm{cm}^{-1}$, and 1-min integration time yields a signal-to-noise of 110 . The minimum detectable change in frost emissivity given this signal-to-noise ratio is 0.03 , corresponding to a change in brightness temperature of 4 $\mathrm{K}$. This assumes that the change in the signal level $(\Delta S)$ of $\Delta S \geq 3 \cdot N E R$ is a reasonable detection criteria, where the noise equivalent radiance $(N E R)$ is defined by $N E R=N E P /\left(\eta A_{\mathrm{m}} \Delta v \Omega_{\mathrm{s}} \sqrt{t}\right)$.

Sensitivity requirements for observations of the outer solar system moons would be significantly more demanding due to the much lower thermal fluxes from these colder planetary bodies. For Titan, the surface temperature is 95 $\mathrm{K}$ and for Triton the surface temperature is $38 \mathrm{~K}$. The peak of the blackbody flux from Triton is 1000 times weaker than the peak flux from Mars. This would indicate that significant improvements in detector sensitivity are required. Conclusions about whether ices on Titan, Triton, and Pluto could be detected using an earth-based instrument will be highly dependent on the assumptions made about future technological capability and on the results of laboratory and theoretical modeling studies, but it appears to be beyond current capability.

\section{CONCLUSION}

Spectral measurements of cloud and surface ices are desirable because their composition can be determined by the identification of unique spectral features associated with a particlular constituent. Cloud or aerosol observations using occultation or narrow-band radiometric techniques tend to undersample the spectrum and therefore often must rely on temperature measurements to aid in determining the composition of ices. Interpretation of near- and mid-infrared observations can be complicated by atmospheric opacity, and in the case of Mars by the presence of significant amounts of suspended dust. The effects of atmospheric gas and dust opacity on martian cloud and surface ice spectra are greatly reduced in the far infrared. The reduced atmospheric effects and the fact that $\mathrm{CO}_{2}$ and $\mathrm{H}_{2} \mathrm{O}$ ices possess unique far-infrared spectral features in the range from 50 to $250 \mathrm{~cm}^{-1}$ are the two principal motivations for investigating the far-infrared spectral properties of cloud and surface ices.

It was found from the laboratory and modeling studies that frost composed of primarily large $\mathrm{H}_{2} \mathrm{O}$ ice particles shows very little spectral variation in the far infrared, making a direct detection of the permanent $\mathrm{H}_{2} \mathrm{O}$ ice cap on
Mars unlikely in this part of the spectrum. Mid-latitude $\mathrm{H}_{2} \mathrm{O}$ ice cloud spectra were shown to be sensitive to cloud ice amount and cloud-surface temperature contrast. The presence of atmospheric dust and gas opacity has a neglible affect on the cloud spectra. Detection of martian midlatitude ice clouds and hazes from an orbiting spacecraft may be possible, but the low brightness contrast between the optically thin far-infrared spectral features and the relatively warm surface background limit this possiblity to clouds having total column ice amounts close to the theoretical upper limit $(\sim 2 \mathrm{pr} \mu \mathrm{m})$ for the martian atmosphere. This conclusion will likely change for the limbviewing geometry available to an orbiting spacecraft. The longer path length and the low background radiance from space should result in a substantially lower detection limit, making the measurement of cloud opacity and cloud temperature possible for a wider range of cloud conditions.

Theoretical calculations of $\mathrm{CO}_{2}$ frost emissivity showed a strong brightness temperature variation of roughly 20 to $30 \mathrm{~K}$ near the 66 and $110 \mathrm{~cm}^{-1}$ lattice bands of solid $\mathrm{CO}_{2}$. Observations of these unique spectral features would allow for an unambiguous detection of the seasonal polar ice caps, both from an orbiting spacecraft and from an earthbased instrument, even in the presence of significant polar hazes and dust. In addition, the high frost emissivity near 66 and $110 \mathrm{~cm}^{-1}$ would suggest that surface temperature could be measured in this region. The low frost emissivity in the region 150 to $500 \mathrm{~cm}^{-1}$ provides better thermal contrast for detecting $\mathrm{H}_{2} \mathrm{O}$ ice cloud spectral features and for measuring the total cloud ice amount. It appears possible to detect the presence of a low-lying $\mathrm{CO}_{2}$ ice haze in the polar region, assuming the underlying frost properties can be properly characterized. Theoretical calculations of $\mathrm{CO}_{2}$ frost emissivity in this spectral region were found to be sensitive to the presence of small amounts of dust or $\mathrm{H}_{2} \mathrm{O}$ ice particles incorporated into the frost. However, the sensitivity of $\mathrm{CO}_{2}$ frost emissivity in this spectral region to other factors, such as model parameters and uncertainty in the imaginary refractive index, would make quantitative measurements of dust and $\mathrm{H}_{2} \mathrm{O}$ ice amounts in the polar cap difficult.

A large primary mirror on the order of 15 to $20 \mathrm{~m}$ in diameter would be needed to reasonably resolve the polar regions on Mars from Earth. NASA's proposed Large Deployable Reflector satellite (Swanson 1991) is an example of the technical feasibility of deploying a far-infrared or submillimeter instrument with a large primary mirror. High angular resolution is also theoretically possible with interferometric techniques involving two or more instruments flown on one or more earth-orbiting satellites. This approach would be particularly attractive for observing ices in the outer solar system where the angular sizes of the icy moons are very small.

Long-term observations of the formation and sublima- 
tion of the seasonal polar caps, as well as the amount of dust incorporated into the ice caps, would provide important information for understanding and modeling the radiative balance in the polar regions and the condensation flow component of the atmospheric circulation on Mars. Analysis of the far-infrared spectral properties of martian cloud and surface ices provides a framework for addressing the feasibility of determining the composition of other planetary ices in the far infrared. Although definite conclusions about the feasibility of observing ices in the outer solar system must await careful laboratory measurements and theoretical modeling, the detection of the thick $\mathrm{NH}_{3}$ and $\mathrm{CH}_{4}$ top-most cloud layers on the giant planets and nonpolar surface ices such as $\mathrm{N}_{2}, \mathrm{CH}_{4}, \mathrm{CO}$, and perhaps $\mathrm{H}_{2} \mathrm{O}$ and $\mathrm{CO}_{2}$ ices, on Triton and Pluto may be possible with the development of very-low-noise, large-aperture or interferometric satellite instruments. The far-infrared spectrum of ices contains useful information regarding composition, cloud opacity, and temperature that, combined with other remote sensing observations, could provide a new diagnostic tool for characterizing planetary ices.

\section{APPENDIX A}

The transmission spectrum, as measured by the FTS instrument, is given as the ratio of the transmission spectrum of the ice film, $I_{\mathrm{SPC}}$, to the blank substrate reference spectrum, $I_{\mathrm{REF}}$,

$$
\begin{aligned}
I_{\mathrm{SPC}} & =T_{\mathrm{W}}^{2} \cdot T_{\mathrm{ice} / \mathrm{sub}} \cdot I_{0} \\
I_{\mathrm{REF}} & =T_{\mathrm{W}}^{2} \cdot T_{\mathrm{sub}} \cdot I_{0},
\end{aligned}
$$

where $T_{\mathrm{W}}^{2}$ is the transmission through the two polyethylene cryostat windows, $T_{\text {sub }}$ is the wedged silicon substrate transmission, and $I_{\mathrm{o}}$ is the source spectrum including the instrument transmission function. $T_{\text {ice/sub }}$ is the transmission of the ice film deposited on the substrate. The measured transmission is then given as

$$
T_{\mathrm{m}}=\frac{I_{\mathrm{SPC}}}{I_{\mathrm{REF}}}=\frac{T_{\mathrm{ice} / \mathrm{sub}}}{T_{\text {sub }}} .
$$

It should be pointed out that the effects of the substrate transmission are not completely removed by the ratio in Eq. (A2) because the reflectivity of the front surface changes with the addition of the ice film. Before analyzing the measurement, $T_{\mathrm{m}}$ was corrected for substrate transmission, $T_{\mathrm{m}}^{\prime}=$ $T_{\mathrm{m}} \cdot T_{\mathrm{sub}}$.

An iterative Kramers-Kronig technique was used to derive the complex refractive index, $\hat{m}=n+i k$, from the measured transmission spectrum. The absorption coefficient was calculated using a Newton iteration scheme,

$$
\alpha_{\mathrm{p}}^{i+1}=\alpha_{\mathrm{p}}^{i}+\left(\ln T_{\text {model }}-\ln T_{\mathrm{m}}^{\prime}\right) / d
$$

where $\alpha_{\mathrm{p}}^{i}$ is the first guess or current estimate of the absorption coefficient, $\alpha_{\mathrm{p}}^{i+1}$ is the next estimate, and $d$ is the film thickness derived from fitting a theoretical thin film model, $T_{\text {model }}$, to interference fringes in the measured spectrum. The thin film model, which is discussed below, is necessary to accurately account for reflection loss and the change in the real part of the refractive index over an absorption band.
The film thickness of $\mathrm{CO}_{2}$ ice was estimated by fitting the theoretical model to the measured transmission spectrum in the region 150 to 600 $\mathrm{cm}^{-1}$. In this spectral region the absorption coefficient is negligibly small and interference fringes are readily observed. The uncertainty in $\mathrm{CO}_{2}$ ice film thickness was estimated to be $3 \%$ using this technique. The use of several different sample thicknesses in the analysis could, in principle, reduce the uncertainty in the derivation of refractive index. However, high-quality thin films of $\mathrm{CO}_{2}$ ice with adequate thickness $(\sim 20$ to 30 $\mu \mathrm{m})$ were very difficult to obtain. It was felt because of this difficulty that uncertainty in scattering and uniformity from film to film would introduce greater uncertainty in the final result. For $\mathrm{H}_{2} \mathrm{O}$ ice, the fitting procedure was complicated by the fact that only very thin films $(d<10$ $\mu \mathrm{m})$ could be used, which results in a rather wide spacing between fringe maxima (or minima) of $\sim 380 \mathrm{~cm}^{-1}$. Therefore, only a single fringe maximum and minimum were observed in the measurement range from 100 to $700 \mathrm{~cm}^{-1}$. In addition, there is measurable absorption over most of the far infrared spectral region which strongly affects the characteristics of the fringe pattern. In many cases, however, fitting the theoretical model to the measured spectrum still allowed a reasonable estimation of film thickness to be made. The derived absorption coefficient for $\mathrm{H}_{2} \mathrm{O}$ ice was compared with data given by Bertie et al. (1969) and a slight adjustment in the estimated film thickness was made for better agreement. This approach resulted in a rather large uncertainty in the determination of $\mathrm{H}_{2} \mathrm{O}$ ice sample thickness of approximately $20 \%$.

Once the film thickness was determined, the absorption coefficient was calculated, using Eq. (A3), by initially setting $n$ to be a constant and $k$ equal to zero in the theoretical model. The imaginary part of the refractive index was then calculated directly from the absorption coefficient, $k=$ $\alpha_{\mathrm{p}} / 4 \pi \nu$, where $v$ is the wavenumber of incident light. The real part of the refractive index was obtained from the Kramers-Kronig dispersion relation given by

$$
n(v)=n_{\infty}+\frac{1}{2 \pi^{2}} \int_{0}^{\infty} \frac{\alpha_{\mathrm{p}}\left(\nu^{\prime}\right) d \nu^{\prime}}{\left(\nu^{\prime 2}-\nu^{2}\right)},
$$

where $n_{\infty}$ is the high frequency refractive index. As can be seen in Eq. (A4), the integrated contributions to the real part of the far-infrared refractive index from higher frequency absorption bands decreases as $1 / \nu^{2}$ and therefore only near- and mid-infrared absorption bands need to be considered in the calculation. For our analysis, the high-frequency refractive index was taken to be the value of the real refractive index of ice at the upper limit of integration. The upper limits were $1000 \mathrm{~cm}^{-1}$ for $\mathrm{CO}_{2}$ ice and $4000 \mathrm{~cm}^{-1}$ for $\mathrm{H}_{2} \mathrm{O}$ ice. The lower limit of integration was $50 \mathrm{~cm}^{-1}$ for $\mathrm{CO}_{2}$ ice and $30 \mathrm{~cm}^{-1}$ for $\mathrm{H}_{2} \mathrm{O}$ ice. A low-frequency correction based on Bertie et al.'s data was used for $\mathrm{H}_{2} \mathrm{O}$ ice since there is still measurable absorption below $100 \mathrm{~cm}^{-1}$. With these upper and lower limits, the truncation error in the calculated far infrared $n(\nu)$ was less than $0.1 \%$ for both ices. Near- and mid-infrared absorption coefficient data used in Eq. (A4) were obtained from Bertie et al. (1969) for water ice and from Warren (1986) for carbon dioxide ice.

We explored the influence of uncertainties in the published near- and mid-infrared absorption coefficients on the calculated far-infrared real refractive indices. Calculation of the far-infrared refractive index using the Kramers-Kronig relation is relatively insensitive to errors in nearand mid-infrared absorption coefficient data. For example, assuming a $20 \%$ error in the published data, the estimated error in the derived real refractive index was less than $3 \%$ for $\mathrm{H}_{2} \mathrm{O}$ ice and $0.5 \%$ for $\mathrm{CO}_{2}$ ice. Systematic error is also introduced into the calculated far-infrared refractive index through uncertainty in the value of $n_{\infty}$. Warren (1986) estimated the uncertainty in $n_{\infty}$ to be \pm 0.05 . Bertie et al. (1969) did not give an error estimate for their derived refractive index, but using Warren as a guide an error of \pm 0.05 was also assumed for $\mathrm{H}_{2} \mathrm{O}$ ice. It should be pointed out that $n_{\infty}$ is temperature dependent, but this dependence was not considered here since other sources of error dominate. 
The iterative procedure using Eqs. (A3) and (A4) was repeated to yield successively better estimates of $\alpha_{\mathrm{p}}, n$, and $k$. In numerical tests using synthetic absorption data, the iterative process converged rapidly to a solution after five iterations, converging to better than $1 \%$ of the true value, well within the instrumental noise.

The theoretical thin film model, used in Eq. (A3), was based on a generalized matrix approach to multi-layered dielectric problems (Born and Wolfe 1970), which allows substrate losses to be explicitly taken into account. The transmission of power through the multi-layered dielectric system is given by

$$
T_{\text {model }}=\frac{n_{\mathrm{L}}}{n_{0}}|t|^{2},
$$

where $n_{\mathrm{o}}$ and $n_{\mathrm{L}}$ are the refractive indices of the first and last mediums, respectively, and $t$ is given by

$$
t=\frac{2 P_{\mathrm{o}}}{\left(m_{11}+m_{12} P_{\mathrm{L}}\right) P_{\mathrm{o}}+\left(m_{21}+m_{22} P_{\mathrm{L}}\right)},
$$

where $P_{i}=\sqrt{\varepsilon_{\mathrm{o}} / \mu_{\mathrm{o}}} \cdot \hat{m}_{i}$ for normally incident light. $\varepsilon_{\mathrm{o}}$ and $\mu_{\mathrm{o}}$ are the vacuum permittivity and permeability, respectively, and $\hat{m}_{i}$ is the complex refractive index of medium $i$. The quantities $m_{i j}$ in Eq. (A6) are elements of the characteristic matrix of the system;

$$
\left[\begin{array}{ll}
m_{11} & m_{12} \\
m_{21} & m_{22}
\end{array}\right]=\left[\begin{array}{ll}
m_{11}^{\prime} & m_{12}^{\prime} \\
m_{21}^{\prime} & m_{22}^{\prime}
\end{array}\right]\left[\begin{array}{ll}
m_{11}^{\prime \prime} & m_{12}^{\prime \prime} \\
m_{21}^{\prime \prime} & m_{22}^{\prime \prime}
\end{array}\right] \cdots,
$$

where $m_{i j}^{\prime}$ and $m_{i j}^{\prime \prime}$ etc. are elements of the individual layer characteristic matrices. For a uniform, lossy dielectric layer of thickness $d_{i}$, the elements of the individual layer characteristic matrix are

$$
\begin{aligned}
& m_{11}^{\prime}=m_{22}^{\prime}=\cosh \left(\gamma_{i} d_{i}\right), \\
& m_{12}^{\prime}=\frac{1}{P_{i}} \sinh \left(\gamma_{i} d_{i}\right), \\
& m_{21}^{\prime}=P_{i} \sinh \left(\gamma_{i} d_{i}\right) .
\end{aligned}
$$

The complex propagation constant is $\gamma_{i}=\alpha_{i}+j \beta_{i}$, where $\alpha_{\mathrm{i}}=2 \pi k_{i} / \lambda_{\mathrm{o}}$ and $\beta_{i}=2 \pi n_{i} / \lambda_{\mathrm{o}}$ and $\lambda_{\mathrm{o}}$ is the vacuum wavelength of incident light.

The generalized matrix approach given above yields a somewhat simpler form for the three-dielectric layer case consisting of vacuum, an ice film of thickness $d_{1}$, and an infinite, lossless substrate. The transmission given by Eq. (A5) becomes

$$
T_{\text {model }}=\frac{n_{\mathrm{L}}}{n_{\mathrm{o}}} \frac{\left|t_{01}\right|^{2}\left|t_{12}\right|^{2} e^{-\gamma_{1} d_{1}}}{\mid 1+r_{01} r_{12} e^{-\left.2 \gamma_{1} d_{1}\right|^{2}}}
$$

with Fresnel reflection and transmission coefficients at the $i-j$ interface given by

$$
t_{i j}=\frac{2 \hat{m}_{i}}{\hat{m}_{i}+\hat{m}_{j}} \quad \text { and } \quad r_{i j}=\frac{\hat{m}_{i}-\hat{m}_{j}}{\hat{m}_{i}+\hat{m}_{j}} .
$$

Equation (A9), or a simplified form of it, has often been used for modeling thin film measurements and implicitly assumes a lossless substrate. This is generally a poor assumption for far-infrared substrate material and therefore the generalized matrix approach was adopted.
The thin film model was modified to accommodate the use of a wedged substrate. Wedging the substrate eliminates interference fringes due to multiple reflections inside the substrate. These unwanted interference fringes are extremely difficult to completely remove from the measured transmission spectrum and may obscure weak absorption bands in the sample. The wedged substrate was modeled using a mean thickness for the substrate and artificially requiring the last medium to have the same refractive index as the substrate but with zero thickness. This eliminates the internal reflection at the substrate-vacuum interface, but also removes a significant surface reflection loss. A correction was made by multiplying the total transmission by the square of the Fresnel transmission coefficient evaluated at the substrate-vacuum interface.

\section{APPENDIX B}

To calculate planetary emission spectra, the full radiative transfer equation must be solved using the appropriate input parameters defined by the atmospheric, cloud, and surface models. The radiative transfer equation for a plane-parallel, azimuthally symmetric atmosphere in local thermodynamic equilibrium can be written

$$
\begin{aligned}
\mu \frac{d I_{\mathrm{v}}(z, \mu)}{d z}= & -\kappa_{\mathrm{ext}} I_{v}(z, \mu)+\frac{\beta_{\mathrm{sca}}}{2} \int_{-1}^{1} \bar{p}\left(\mu, \mu^{\prime}\right) I_{\mathrm{v}}\left(z, \mu^{\prime}\right) d \mu^{\prime} \\
& +\left(\kappa_{\mathrm{gas}}+\beta_{\mathrm{abs}}\right) B_{\mathrm{v}}(T),
\end{aligned}
$$

where $I_{\nu}(z, \mu)$ is the monochromatic intensity at frequency, $\nu$, at a height above the surface, $z$, and in the zenith direction, $\theta$, where $\mu=\cos \theta$. The total extinction coefficient, $\kappa_{\text {ext }}$, is the sum of gaseous absorption, $\kappa_{\text {gas }}$, particle absorption, $\beta_{\mathrm{abs}}$, and particle scattering, $\beta_{\mathrm{sca}}$. The integral term on the right-hand side of Eq. (B1) represents the intensity that is scattered into direction $\mu$ by cloud and dust particles. The particle phase function $\bar{p}\left(\mu, \mu^{\prime}\right)$ is the cross-section-weighted average of the individual phase functions for each particle type (e.g., atmospheric dust or $\mathrm{H}_{2} \mathrm{O}$ ice). The phase functions for the individual particle types were averaged over their respective size distributions. Both the particle cross sections and phase functions for a given particle size were calculated using Mie's theory. The thermal source term is given by the Planck function, $B_{\mathrm{v}}(T)=2 \mathrm{hc}$ / $\nu^{3}(\exp (h v / k T)-1)^{-1}$.

The solution to Eq. (B1) for $z \rightarrow \infty$, subject to the appropriate boundary conditions, yields the total outgoing intensity at the top of the atmosphere, taken to be $120 \mathrm{~km}$ in this model. The upper boundary condition assumes the incident intensity is zero. The lower boundary condition at $z=0$ includes the reflected downward directed atmospheric intensity, $I^{-}\left(0, \mu^{\prime}\right)$, and thermal emission from the surface, $\varepsilon(\mu)$ :

$$
I_{\mathrm{S}}(0, \mu)=2 \int_{0}^{1} \rho\left(\mu, \mu^{\prime}\right) \mu^{\prime} I^{-}\left(0, \mu^{\prime}\right) d \mu^{\prime}+\varepsilon(\mu)
$$

The bi-directional reflectance, $\rho\left(\mu, \mu^{\prime}\right)$, depends on both the incident direction, $\mu^{\prime}$, and on the reflected direction, $\mu$, and in general it is a complicated function of both directions. Hapke's surface model, which is used to calculate $\rho\left(\mu, \mu^{\prime}\right)$ and $\varepsilon(\mu)$, is discussed below. Using the average radiation field to approximate the radiation incident on the surface from all directions, Eq. (B2) becomes

$$
I_{\mathrm{s}}(0, \mu) \approx 2 \bar{I}^{-}(0) \int_{0}^{1} \rho\left(\mu, \mu^{\prime}\right) \mu^{\prime} d \mu^{\prime}+\varepsilon(\mu)
$$

where the average radiation field was evaluated using a two-stream approximation. For a uniform incident radiation field, the integration over $\mu^{\prime}$ in Eq. (B3) yields the hemispherical-directional spectral reflectivity, written $\bar{\rho}(\mu)=1-\varepsilon(\mu)$. Equation (B3) then becomes approximately $I_{\mathrm{s}}(0, \mu) \approx \bar{\rho}(\mu) \bar{I}^{-}(0)+\varepsilon(\mu)$. 
The approach used to solve the radiative transfer equation was to separate the monochromatic intensity into two components, a reduced (or direct) intensity component due to atmospheric and surface emission, $I_{r i}(z, \mu)$, and a diffuse intensity component due to scattering by atmospheric particles, $I_{d}(z, \mu)$. The total intensity, $I(z, \mu)=I_{r i}(z, \mu)+I_{\mathrm{d}}(z$, $\mu)$ must satisfy Eq. (B1). Substituting this expression into Eq. (B1) yields a set of two coupled equations

$$
\begin{aligned}
\mu \frac{d I_{r i}(z, \mu)}{d z}= & -\kappa_{\mathrm{ext}} I_{r i}(z, \mu)+\left(\kappa_{\mathrm{gas}}+\beta_{\mathrm{abs}}\right) B_{v}(T), \\
\mu \frac{d I_{\mathrm{d}}(z, \mu)}{d z}= & -\kappa_{\mathrm{ext}} I_{d}(z, \mu) \\
& +\frac{\beta_{\mathrm{sca}}}{2} \int_{-1}^{1} \bar{p}\left(\mu, \mu^{\prime}\right)\left(I_{r i}(z, \mu)+I_{d}(z, \mu)\right) d \mu^{\prime} .
\end{aligned}
$$

The atmosphere was divided into 30 layers, each $4 \mathrm{~km}$ thick. The reduced intensity equation (Eq. (B4a)) was numerically integrated using a four-point Gaussian quadrature over each layer. The combination of layer thickness and the number of quadrature points yielded a numerical error in the outgoing intensity of less than $4 \%$. A line-by-line technique was used to calculate the reduced intensity at each level in the atmosphere, summing contributions from all the far-infrared transition lines of $\mathrm{CO}$, $\mathrm{H}_{2} \mathrm{O}, \mathrm{O}_{2}$, and $\mathrm{O}_{3}$. The main atmospheric constituents $\mathrm{CO}_{2}$ and $\mathrm{N}_{2}$ do not contribute to the far-infrared spectrum because these symmetric molecules do not possess permanent dipole moments and therefore exhibit no pure rotational transitions. Spectral data for $\mathrm{CO}, \mathrm{H}_{2} \mathrm{O}, \mathrm{O}_{2}$, and $\mathrm{O}_{3}$ were taken from the HITRAN database (Rothman et al. 1987). Spinpartition function data were obtained from the JPL Spectral Line Catalog (Poynter and Pickett 1985). Lineshape was modeled using a Voigt line profile (Drayson 1976). A fine frequency spacing was used near each line center, which adequately samples the narrow pressure-broadened lines in the martian atmosphere. A coarse spacing was used in the far wings and continuum region.

Radiation scattered by cloud and dust particles was calculated using a successive orders of scattering approximation (Hansen and Travis 1974). This approach has the advantage of handling non-homogeneous layers and it provides greater physical insight into the scattering process than most other methods. A low-order scattering approximation is sufficient for the long wavelengths of the far infrared and the small particle sizes present in the martian atmosphere. The disadvantage of this method is that it can be very slow to converge for cases where the single scattering albedo of the particle is close to unity and for optically thick clouds. Generally, the single scattered diffuse intensity was less than about $1 \%$ of the total intensity for mid-latitude clouds and moderately dusty conditions, indicating that scattering can be neglected in these cases. In the case of the polar hood condensates, calculations showed that approximately $15 \%$ of the total outgoing intensity was due to the single scattered diffuse intensity, primarily due to scattering by the relative large $\mathrm{CO}_{2}$ ice haze particles.

A particulate surface model developed by Hapke (1993) was used to calculate reflection and emission from the martian regolith and polar caps. Hapke's model is an approximate analytic solution to the radiative transfer equation that allows composition, particle size, packing density, and a subsurface temperature gradient to be explicitly included. A mixture of particle types, such as dust and $\mathrm{CO}_{2}$ frost, can also be considered. The model assumes the surface is composed of particles much larger than the wavelength of interest and that particles are irregularly shaped and randomly oriented. The large particle approximation allows the scattering cross section of a particle to be calculated using the geometric optics approximation. The large particle assumption may not be a particularly good one for the long wavelengths considered here, although grain sizes for the residual caps may be quite large (Calvin 1990, Kieffer 1990).
Hapke's model does provide a useful means for obtaining a first-order estimate of the far-infrared spectral characteristics of planetary frosts. The emitted radiance from a particulate surface is given by Hapke (1993):

$$
\varepsilon(\mu)=\frac{B_{\mathrm{o}}}{\pi} \gamma H(w, \mu)+\frac{B_{1}}{\pi} \frac{L}{L+\mu} \gamma^{2} H(w, \mathrm{~L}) H(w, \mu) .
$$

The albedo factor is defined by $\gamma=\sqrt{1-\omega_{\mathrm{o}}}$ where $\omega_{\mathrm{o}}$ is the average single scattering albedo for the medium. The Planck blackbody function was parameterized by Hapke to allow a subsurface temperature gradient to be included and still yield a convenient closed-form solution. The Planck function is given by $B(\zeta)=B_{\mathrm{o}}+B_{1} \exp (-|\zeta| / \Lambda)$, where $\zeta$ is the distance in the medium below the surface and $\Lambda$ is a "thermal emission scale height" given by $L=E \Lambda$. $E$ is the extinction coefficient of the medium and $L$ is a dimensionless length scale. $H(w, L)$ and $H(w, \mu)$ are Chandrasekhar's $H$-functions (Chandrasekhar 1960):

$$
H(w, x)=1+\frac{w}{2} x H(w, x) \int_{0}^{1} \frac{H(w, x)}{(x+y)} d y .
$$

Hapke gives an approximation to Chandrasekhar's $H$-functions which he estimates to give less than $4 \%$ error,

$$
H(w, \mu) \approx 1+\frac{1+2 \mu}{1+2 \gamma \mu} \text { and } H(w, L) \approx 1+\frac{1+2 L}{1+2 \gamma L} .
$$

The hemispherical-directional reflectivity of a particulate surface is related to the directional emissivity by $\bar{\rho}(\mu)=1-\varepsilon(\mu)$.

\section{ACKNOWLEDGMENTS}

This research was supported in part by funds provided by the NASA/ Center for Space Terahertz Technology at the University of Michigan, and by NASA Planetary Atmospheres Program Grant NAGW-1771 to S.K.A. B.R.J. acknowledges partial support from the National Center for Atmospheric Research during the completion of this work. The National Center for Atmospheric Research is sponsored by the National Science Foundation. Finally, the authors thank the anonymous Reviewers for their careful review of this manuscript.

\section{REFERENCES}

Atreya, S. K. 1986. Atmospheres and Ionospheres of the Outer Planets and Their Satellites. Springer-Verlag, New York.

Atreya, S. K., J. B. Pollack, And M. S. Matthews (Eds.) 1989. Origin and Evolution of Planetary and Satellite Atmospheres. Univ. of Arizona Press, Tucson.

Atreya, S. K. AND Z. G. Gu 1994. Stability of the martian atmosphere: Is heterogeneous catalysis essential? J. Geophys. Res. 99, 13,133-13,145.

Barth, C. A. 1974. The atmosphere of Mars. Annu. Rev. Earth Planet. Sci. 2, 333-367.

Bertie, J. E., H. J. Labbé, And E. Whalley 1969. Absorptivity of ice I in the range 4000 to $30 \mathrm{~cm}^{-1}$. J. Chem. Phys. 50, 4501-4520.

Born, M., And E. Wolf 1970. Principles of Optics. Pergamon, Oxford.

BRiggs, G. A, And C. B. Leovy 1974. Mariner 9 observations of the Mars north polar hood. Bull. Am. Meteor. Soc. 55, 278-296.

Brown, K. G., AND W. T. KING 1970. Infrared intensities of the lattice modes of solid carbon dioxide. J. Chem Phys. 52, 4437-4440. 
Calvin, W. M. 1990. Additions and corrections to the absorption coefficients of $\mathrm{CO}_{2}$ ice: Applications to the martian south polar cap. $J$. Geophys. Res. 95, 14,743-14,750.

Chandrasekhar, S. 1960. Radiative Transfer, p. 105. Dover, New York.

Chassefièrre, E., J. E. Blamont, V. A. Krasnopolosky, O. I. KoraBlev, S. K. Atreya And R. A. West 1992. Vertical structure and size distributions of martian aerosols from solar occultation measurements. Icarus 97, 46-69.

Christensen, P. R., And R. W. Zurek 1984. Martian north polar hazes and surface ices: Results from the Viking survey/completion mission. J. Geophys. Res. 89, 4587-4596.

Curran, R. J., B. J. Conrath, R. A. Hanel, V. G. Kunde, and J. C. Pearl 1973. Mars: Mariner 9 spectroscopic evidence for $\mathrm{H}_{2} \mathrm{O}$ ice clouds. Science, 182, 381-383.

Drayson, R. 1976. Rapid computation of the Voigt profile. J. Quant. Spectrosc. Radiat. Transfer 16, 611-614.

FAlk, M. 1987. Amorphous solid carbon dioxide. J. Chem. Phys. 86, $560-564$

FINK, U., AND G. Sill 1982. The infrared spectral properties of frozen volatiles, In Comets, (L. L. Wilkening, Ed.), Univ. of Arizona Press, Tucson.

Hansen, G. B. 1993. The spectral absorption of $\mathrm{CO}_{2}$ ice in the thermal infrared. Bull. Am. Astron. Soc. 25, 1003.

Hansen, J. E., And L. D. Travis 1974. Light scattering in planetary atmospheres. Space Sci. Rev. 16, 527-610.

HAPKe, B. 1993. Combined theory of reflectance and emittance spectroscopy, In Remote Geochemical Analyses, (C. Pieters and P. Englert, Eds.), Cambridge Univ. Press, Cambridge, UK.

Hudgins, D. M., S. A. SAndford, L. J. Allamandola, And A. G. G. M. TIELENS 1993. Mid- and far-infrared spectroscopy of ices: Optical constants and integrated absorbances. Astrophys. J. Suppl. 86, 713-870.

Hunt, G. E. 1980. On the infrared radiative properties of $\mathrm{CO}_{2}$ ice clouds: Application to Mars. J. Geophys. Res. 85, 481-484.

Hunt, G. R., AND R. K. VinCENT 1968. The behavior of spectral features in the infrared emission from particulate surfaces of various grain sizes. J. Geophys. Res. 73, 6039-6046.

Hunten, D. M. 1969. The upper atmosphere of Jupiter. J. Atmos. Sci. 26, 826-834.

JAkosky, B. M. 1985. The seasonal cycle of water on Mars. Space Sci. Rev. 41, 131-200.

JAKOSKY, B. M. AND C. B. FARMER 1982. The seasonal and global behavior of water vapor in the martian atmosphere: Complete global results of the Viking atmospheric water detector experiment. J. Geophys. Res. 87, 2999-3019.

James, P. B., AND G. R. North 1982. The seasonal $\mathrm{CO}_{2}$ cycle on Mars: An application of an energy balance climate model. J. Geophys. Res. 87, 10,271-10,283.

KAHN, R. 1990. Ice haze, snow and the Mars water cycle. J. Geophys. Res. 95, 14,677-14,693.

KHANnA, R. K., M. J. OspinA, AND G. ZHAO 1988. Infrared band extinctions and complex refractive indices of crystalline $\mathrm{C}_{2} \mathrm{H}_{2}$ and $\mathrm{C}_{4} \mathrm{H}_{2}$. Icarus 73, 527-535.

Khare, B. N., W. R. Thompson, C. Sagan, E. T. Arkawa, C. Bruel, J. P. Judish, R. K Khanna, And J. B. Pollack 1990. Optical constants of solid methane, In First International Conference on Laboratory Research for Planetary Atmospheres, (K. Fox, J. E. Allen, L. J. Stief, and D. T. Quillen, Eds.), NASA Conf. Publications, Vol. 3077, pp. 327-339. National Aeronautics \& Space Admin., Washington, DC.
Kieffer, H. M., P. R. Christensen, T. Z. Martin, E. D. Miner, And F. D. PAlluCONi 1976. Temperatures of the martian surface and atmosphere: Viking observations of diurnal and geometric variations. Science 194, 1346-1351.

KIEFFER, H. H. 1990. $\mathrm{H}_{2} \mathrm{O}$ grain size and amount of dust in Mars' residual north polar cap. J. Geophys. Res. 95, 1481-1493.

KuAn, T. S. 1969. I. Lattice Vibrations of Solid $\alpha$-Nitrogen and AtomAtom Intermolecular Potential. II. Intensities of the Far Infrared Absorption Lines of Solid Carbon Dioxide. Ph.D. Thesis, University of Southern California, Los Angeles.

Lindal, G. F., H. B. Hotz, D. N. Sweetnam, Z. Shippony, J. P. Brenkle, G. V. Hartsell, R. T. Spear, and W. H. Michael 1979. Viking radio occultation measurements of the atmosphere and topography of Mars: Data acquired during one martian year of tracking. J. Geophys. Res. 84, 8443-8456.

Masterson, C. M. and R. K. Khanna 1990. Absorption intensities and complex refractive indices of crystalline $\mathrm{HCN}, \mathrm{HC}_{3} \mathrm{~N}$, and $\mathrm{C}_{2} \mathrm{~N}_{2}$ in the infrared region. Icarus $\mathbf{8 3}, 83-92$.

McCREIGHT, C. 1991. Report of the direct infrared sensors panel. In ASTROTECH 21 Workshop Proceedings: Sensor Systems for Space Astrophysics in the 21st Century, JPL Publication 91-24, Vol. 2, pp. 34-49.

Moersch, J. E., And P. R. Christensen 1995. Thermal emission from particulate surfaces: A comparison of scattering models with measured spectra. J. Geophys. Res. 100, 7465-7477.

Mukai, T. AND C. KoIKe 1990. Optical constants of olivine particles between wavelengths of 7 and $200 \mu \mathrm{m}$. Icarus 87, 180-187.

Owen, T., K. Biemann, D. R. Rushneck, J. E. Biller, D. W. Howarth, AND A. L. LAfleur 1977. The composition of the atmosphere at the surface of Mars. J. Geophys. Res. 82, 4635-4639.

Paige, D. A., And A. P. Ingersoll 1985. Annual heat balance of the martian polar caps: Viking observations, Science 228, 1160-1168.

Pearl, J., M. NGOH, M. Ospina And R. Khanna 1991. Optical constants of solid methane and ethane from 10,000 to $450 \mathrm{~cm}^{-1}$. J. Geophys. Res. 96, 17,477-17,482.

Pollack, J. B., D. Colburn, R. Kahn, J. Hunter, W. Van Camp, C. E. Carlston, And M. R. Wolf 1977. Properties of aerosols in the martian atmosphere, as inferred from Viking Lander imaging data. $J$. Geophys. Res. 82, 4479-4496.

Pollack, J. B., R. M. Haberle, R. M. Schaeffer, and H. Lee 1990. Simulations of the general circulation of the martian atmosphere. I. Polar processes. J. Geophys. Res. 95, 1447-1473.

Pollack, J. B., O. B. Toon, And B. N. Khare 1997. Optical properties of some terrestrial rocks and glasses. Icarus 19, 372-389.

Poynter, R. L., And H. M. Pickett 1985. Submillimeter, millimeter, and microwave spectral line catalog. Appl. Opt. 24, 2235-2240.

Ron, A., AND O. SCHNEPP 1967. Lattice vibrations of the solids $\mathrm{N}_{2}, \mathrm{CO}_{2}$, and CO. J. Chem. Phys. 46, 3991-3998.

Rossow, W. B. 1978. Cloud microphysics: Analysis of the clouds of Earth, Venus, Mars, and Jupiter. Icarus 36, 1-50.

Rothman, L. S., R. R. Gamache, A. Goldman, L. R. Brown, R. A. Troth, H. M. Pickett, R. L. Poynter, J. M. Flaud, C. Camy-Peyret, A. Barbe, N. Husson, C. P. Rinsland, and M. A. H. Smith 1987. The HITRAN database: 1986 edition. Appl. Opt. 26, 4058-4097.

Salama, F., L. J. Allamandola, F. C. Witteborn, D. P. Cruikshank, S. A. SANDFord, AND J. D. Bregman 1990. The 2.5-5.0 $\mu \mathrm{m}$ spectra of Io: Evidence for $\mathrm{H}_{2} \mathrm{~S}$ and $\mathrm{H}_{2} \mathrm{O}$ frozen in $\mathrm{SO}_{2}$. Icarus 83, 66-82.

SANTEE, M., AND D. CRISP 1993. Thermal structure and dust loading of the martian atmosphere during late southern summer: Mariner 9 revisited. J. Geophys. Res. 98, 3261-3279. 
Schwartz, Y. A., A. Ron, AND S. Kimel 1971. Far-infrared spectra of ethane, ethylene and acetylene. J. Chem. Phys. 54, 99-105.

Sill, G., U. FinK, AND J. R. Ferraro 1980. Absorption coefficients of solid $\mathrm{NH}_{3}$ from 50 to $7000 \mathrm{~cm}^{-1}$. J. Opt. Soc. Am. 70, 724-739.

SINGER, R. B. 1982. Spectral evidence for the mineralogy of high albedo soils and dust on Mars. J. Geophys. Res. 87, 10,159-10,168.

Soderblom, L. A. 1993. The composition and mineralogy of the martian surface from spectroscopic observations: $0.3 \mu \mathrm{m}$ to $50 \mu \mathrm{m}$, In Mars (H. H. Kieffer, B. M. Jakosky, C. W. Snyder, and M. S. Matthews, Eds.), Univ. of Arizona Press, Tucson.

Swanson, P. N. 1991. The next century astrophysics program, In ASTROTECH 21 Workshop Proceedings: Sensor Systems for Space Astrophysics in the 21st Century, JPL Publication 91-24, Vol. 2, pp. 7-14.
TAUB, W. A., AND M. T. STIER 1976. Theoretical atmospheric transmission in the mid- and far-infrared at four altitudes. Appl. Opt. 15, 364-377.

Toon, O. B., J. B. Pollack, W. Ward, J. A. Burns, and K. Bilski 1980. The astronomical theory of climatic change on Mars. Icarus 44, 552-607.

Toon, O. B., J. B. Pollack And C. Sagan 1977. Physical properties of the particles composing the martian dust storms of 1971-1972. Icarus 30, 663-696.

Warren, S. G. 1986. Optical constants of carbon dioxide ice. Appl. Opt. 25, 2650-2674.

Warren, S. G., W. J. Wiscombe and J. F. Firestone 1990. Spectral albedo and emissivity of $\mathrm{CO}_{2}$ in martian polar caps: Model results. $J$. Geophys. Res. 95, 14,717-14,741. 